\title{
Entropy Condition Satisfying Approximations for the Full Potential Equation of Transonic Flow*
}

\author{
By Stanley Osher, Mohamed Hafez and Woodrow Whitlow, Jr.
}

\begin{abstract}
We shall present a new class of conservative difference approximations for the steady full potential equation. They are, in general, easier to program than the usual density biasing algorithms, and in fact, differ only slightly from them. We prove rigorously that these new schemes satisfy a new discrete "entropy inequality", which rules out expansion shocks, and that they have sharp, steady, discrete shocks. A key tool in our analysis is the construction of an "entropy inequality" for the full potential equation itself. We conclude by presenting results of some numerical experiments using our new schemes.
\end{abstract}

I. Introduction. The full potential equation is a common model for describing supersonic and subsonic flow close to the speed of sound. The flow is assumed to be that of a perfect gas, and the assumptions of irrotationality and constant entropy are made. The resulting equation is a single nonlinear partial differential equation of second order, which changes type from hyperbolic to elliptic, as the flow goes from supersonic to subsonic. Flows with a supersonic component generally have solutions with shocks, so the conservation form of the equation is important.

This formulation, (FP), is one of three conservative formulations used for inviscid transonic flows. The other two are transonic small-disturbance equation, (TSD), and Euler equation, (EU), which is the exact inviscid formulation. The FP formulation is the most efficient of the three in terms of accuracy-to-cost ratio for a wide range of inviscid transonic flow applications for real geometries. TSD is valid for thin wings at free stream Mach numbers near unity, and EU, while the least restrictive, involves the most complicated system of equations.

During the last few years, many numerical calculations using FP have been presented, e.g., [19], [14], [17], and [6]. The object of our present investigation is twofold. First, we wish to put the theory of nonlinear difference approximations to FP on a sound theoretical basis, via an "entropy condition", as described below. Second, we introduce a new class of entropy condition satisfying approximations, which are, in general, no more complicated to program than the usual density

Received July 15, 1983.

1980 Mathematics Subject Classification. Primary 65M10; Secondary 76H05, 65M05.

Key words and phrases. Full potential equation, transonic flow, entropy condition, difference approximations.

*Research supported by NASA Grant \# NAG1-273, NSF Grant \# MCS 82-00788, and ARO Grant \#29-82-K0090. 
biasing algorithms, and in fact, differ only slightly from them. These new algorithms, besides having a solid (nonlinear) mathematical basis, also seem to outperform the existing algorithms numerically.

In 1980, Engquist and Osher [8] introduced entropy condition satisfying approximations for TSD. They constructed a scheme, which is a simple modification of the commonly used Murman algorithm, [21], and proved that their scheme satisfied an entropy inequality for TSD. Murman's algorithm was earlier shown to violate the entropy condition, [18], and to have stable expansion shock solutions [8], [29]. In [13], Goorjian and Van Buskirk incorporated the E-O scheme into an existing TSD code, using only minor coding modifications to change from the standard Murman algorithm. For steady flows, the convergence is more robust and about $35 \%$ faster. For unsteady flows, the allowable time step is around 30 times larger.

The steady profiles for both methods are very similar, except that nonlinear instabilities were triggered using Murman's scheme for a blunt airfoil, while the E.O. method converged with no problems.

For unsteady flows, the new method allowed time steps at least an order of magnitude larger, but, perhaps more importantly, one case illustrated the following phenomenon.

The Murman scheme can trigger transient numerical instabilities; although these instabilities will not cause calculations to diverge, they will cause large errors in the pressure profiles. Many users of these codes, such as aeroelasticians, are particularly interested in integrated quantities such as the unsteady aerodynamic loads. These users could be unaware of these large errors, unless they monitored, in addition, the calculation of the pressure coefficients.

Additional experiments were performed using the E.O. algorithm for TSD by Edwards, et al. [7]. There, they were able to calculate large amplitude motion and large angles of attack. Thus, transonic flutter solutions, which could previously not even be calculated using existing production codes, were obtained, and found to be quite accurate.

A great deal of nonlinear analysis has recently been used to analyze and construct conservation form approximations to hyperbolic systems of conservation laws, e.g., [23], [16], [24], [25], and [28]. Several successful "high resolution" schemes for EU have been constructed, and complex flows with strong shocks have been computed: [4], [16], [2], and [23]. This type of analysis is not directly applicable to FP, for reasons described in Section II.

The format of our paper is as follows. Section II is purely analytic, i.e., nonnumerical. There, after a preliminary description of the properties of FP, we discuss the concept of the entropy condition, and construct a new entropy condition for FP.

[Note added in proof. We have recently learned that the entropy condition, (Theorem 2.1) below, was first obtained (using a different method) in [26]. We are grateful to the authors of that work for bringing this to our attention.] This inequality is enforced across a shock for FP if and only if the usual criterion of Mach number decreasing across a shock is valid: Theorem (2.1). We also explain why these new ideas are needed, i.e., lack of strict hyperbolicity for the unsteady FP. In Section III, we construct difference approximations for FP based on the concept of E schemes, introduced in [22] for scalar conservation laws. We show rigorously that these 
schemes admit only physically correct limit solutions to FP (Theorem (3.1)). In Section IV, we give examples of our new class of approximations involving the E.O. scheme, Godunov's scheme [11], and general fixes of Murman's scheme [29]. The concept of flux biasing is presented, and shown to be simpler computationally than the usual density biasing, as well as having the same truncation error. This section is the most important for our more applied readers, because the algorithms are presented there. Section V contains the proof of Theorem 3.1 by obtaining a discrete version: inequality (5.3). Finally, Section VI gives the results of numerical experiments showing the worth of the algorithm, based on E.O., in body fitted coordinates. We shall call this version the Hafez-Osher, or H.-O. algorithm in a parallel work, [15], where more numerical evidence is given, and in future work on this subject.

Some earlier attempts have been made to extend the ideas of [8] from TSD to FP. See Goorjian et al. [12], and Boerstoel [1]. Indeed, Boerstoel used flux biasing to construct an algorithm for FP in general coordinate systems, formally extending E.O. from TSD to FP.

II. An Entropy Inequality for the Full Potential Equation. We consider the differential equation

$$
(\rho u)_{x}+(\rho u)_{y}=0
$$

where the density, $\rho>0$, is defined through Bernoulli's law

$$
\frac{q^{2}}{2}+\frac{a^{2}}{\gamma-1}=\frac{1}{M_{\infty}^{2}(\gamma-1)}+\frac{1}{2}
$$

Here

$$
q=\sqrt{u^{2}+v^{2}}<q_{\max }=\sqrt{1+\left(\frac{2}{\gamma-1}\right) M_{\infty}^{-2}}, \quad a=\frac{\rho^{(\gamma-1) / 2}}{M_{\infty}}
$$

are the absolute speed and sound speed, respectively.

The constants

$$
\gamma \approx 1.4, \quad M_{\infty}>0
$$

are given.

The flow is potential, which means there exists a scalar function $\Phi$, with

$$
u=\Phi_{x}, \quad v=\Phi_{y}
$$

even across discontinuities.

$$
u_{y}=v_{x}
$$

(2.1)-(2.4) yield a hyperbolic or elliptic equation, depending upon the Mach number $M=q / a$. We have

$$
\begin{aligned}
& M>1 \Leftrightarrow \text { supersonic flow } \Leftrightarrow \text { hyperbolic, } \\
& M<1 \Leftrightarrow \text { subsonic flow } \Leftrightarrow \text { elliptic. }
\end{aligned}
$$

It turns out that

$$
M>1 \Leftrightarrow q>q^{*}=a^{*}
$$

with the sonic value

$$
q^{*}=\sqrt{\frac{M_{\infty}^{-2}+(\gamma-1) / 2}{(\gamma+1) / 2}} .
$$


We wish to solve (2.1)-(2.4) by obtaining it as the steady (time-independent) solution to an unsteady hyperbolic system of conservation laws, endowed with a convex entropy, in the sense of Lax [20]. This entropy is not to be confused with the true, physical, entropy of gas dynamics, and will be described below.

Such a system can be written as

$$
w_{t}+f(w)_{x}+g(w)_{y}=0,
$$

where

$$
w=\left(w_{1}, \ldots, w_{d}\right)^{T}, \quad f=\left(f_{1}(w), \ldots, f_{d}(w)\right)^{T}, \quad g=\left(g_{1}(w), \ldots, g_{d}(w)\right)^{T} .
$$

The Jacobian matrices

$$
A=\left\{\frac{\partial f_{i}}{\partial w_{j}}\right\}, \quad B=\left\{\frac{\partial g_{i}}{\partial w_{j}}\right\}
$$

are such that any nontrivial real linear combination of $A$ and $B$ has real eigenvalues and a complete set of eigenvectors.

Many of the equations of physics of this type are endowed with an additional convex conservation law. This means that there usually exists a scalar, convex, function $V(w)$, which, for smooth solutions $w$ of (2.6), satisfies

$$
V_{t}+F_{x}+G_{y}=0 \text {. }
$$

$V$ is called the entropy, and $F, G$ are the associated entropy fluxes.

For a list of physical equations and corresponding entropies, see [5].

Nonsmooth weak solutions of (2.6) are not unique. We require, in addition, that they be the limit, as $\varepsilon \downarrow 0$, of solutions to the regularized equation

$$
w_{t}^{\varepsilon}+f\left(w^{\varepsilon}\right)_{x}+g\left(w^{\varepsilon}\right)_{y}=\varepsilon\left(w_{x x}^{\varepsilon}+w_{y y}^{\varepsilon}\right) .
$$

Lax has shown, [20], that a necessary condition for this to be true is that the entropy inequality (in the sense of distributions)

$$
V_{t}+F_{x}+G_{y} \leqslant 0
$$

be satisfied.

We now assume that $A$ has distinct eigenvalues

$$
\lambda_{1}(w)<\lambda_{2}(w)<\cdots<\lambda_{d}(w),
$$

with associated right eigenvectors $r_{1}(w), \ldots, r_{d}(w)$. A characteristic $k$-field is called genuinely nonlinear if

$$
\nabla_{w} \lambda_{k} \cdot r_{k} \neq 0
$$

for all $w$.

Suppose the weak solution to (2.6) is piecewise continuous with a point of discontinuity. We denote by $w^{L}, w^{R}$, the values on the left respectively right, sides of the discontinuity. Such a point of discontinuity is a $k$-shock if both

(a) The Rankine-Hugoniot relation

$$
s\left(w^{L}-w^{R}\right)=f\left(w^{L}\right)-f\left(w^{R}\right),
$$

for $s$ the speed of propagation of the shock, holds; and 
(b) there are exactly $k-1$ of the characteristic speeds $\lambda_{\nu}\left(w^{L}\right)<s$, and $d-k$ speeds $\lambda_{\nu}\left(w^{R}\right)>s$,

$$
\lambda_{k-1}\left(w^{L}\right)<s<\lambda_{k}\left(w^{L}\right), \quad \lambda_{k}\left(w^{R}\right)<s<\lambda_{k+1}\left(w^{R}\right) .
$$

This is the (geometric) shock condition for systems. Lax [20] showed for genuinely nonlinear characteristic fields, that, for weak $k$-shocks, the shock condition is equivalent to the entropy inequality, which in this case becomes

$$
s\left(V\left(w^{L}\right)-V\left(w^{R}\right)\right)-F\left(w^{L}\right)+F\left(w^{R}\right)<0 .
$$

We shall use this apparatus to solve (2.1)-(2.4). A first attempt might be to consider the unsteady full potential equation

$$
\rho_{t}+(\rho u)_{x}+(\rho u)_{y}=0
$$

together with the unsteady Bernoulli equation

$$
\frac{u^{2}+v^{2}}{2}+\frac{a^{2}}{\gamma-1}+\Phi_{t}=k(t) .
$$

Taking the space gradient of (2.16) leads us to a system of three conservation laws

$$
\begin{aligned}
\left(\begin{array}{l}
\rho \\
u \\
v
\end{array}\right)_{t} & +\left(\begin{array}{c}
\rho u \\
\frac{1}{2}\left(u^{2}+v^{2}\right)+a^{2} /(\gamma-1) \\
0
\end{array}\right)_{x} \\
& +\left(\begin{array}{c}
\rho v \\
0 \\
\frac{1}{2}\left(u^{2}+v^{2}\right)+a^{2} /(\gamma-1)
\end{array}\right)_{y}=\left(\begin{array}{l}
0 \\
0 \\
0
\end{array}\right) .
\end{aligned}
$$

Unfortunately, this system is not strictly hyperbolic. The eigenvalues of the matrix

$$
A \zeta+B \eta,
$$

for $\zeta, \eta$ real, with $\zeta^{2}+\eta^{2}=1$, are $\hat{u}-a, 0$, and $\hat{u}+a$, for

$$
\hat{u}=u \zeta+v \eta
$$

When $\hat{u}=a$, or $\hat{u}=-a$, the resulting matrix is not diagonalizable. This means, computationally, that any numerical vorticity which develops, will necessarily generate a numerical instability. This causes problems for numerical methods based on this approach [3].

Instead, we consider the following system, motivated by [29],

$$
-\rho_{t}=(\rho q \cos \theta)_{x}+(\rho q \sin \theta)_{y}, \quad \theta_{t}=-(q \sin \theta)_{x}+(q \cos \theta)_{y},
$$

where $-\pi<\theta \leqslant \pi$ is defined through

$$
u=q \cos \theta, \quad v=q \sin \theta,
$$

and $\rho=\rho(q)$ is defined through Bernoulli's law, (2.2),

$$
\rho=\left(1-\frac{\gamma-1}{2} M_{\infty}^{2}\left(q^{2}-1\right)\right)^{1 /(\gamma-1)}
$$

with

$$
\frac{d}{d q} \rho=\frac{-\rho q}{a^{2}}<0
$$


Thus $-\rho$ is an increasing function of $q$, and vice versa.

If we write $(2.18)$ as

$$
E W_{t}+A W_{x}+B W_{y}=0
$$

with

$$
W=\left(\begin{array}{l}
q \\
\theta
\end{array}\right)
$$

then

$$
\begin{gathered}
E=\left[\begin{array}{cc}
\frac{\rho q}{a^{2}} & 0 \\
0 & 1
\end{array}\right], \quad-A=\left[\begin{array}{cc}
\rho\left(1-\left(\frac{q^{2}}{a^{2}}\right)\right) \cos \theta & -\rho q \sin \theta \\
-\sin \theta & -q \cos \theta
\end{array}\right], \\
-B=\left[\begin{array}{cc}
\rho\left(1-\frac{q^{2}}{a^{2}}\right) \sin \theta & \rho q \cos \theta \\
\cos \theta & -q \sin \theta
\end{array}\right] .
\end{gathered}
$$

If we then multiply $(2.22)$ by the diagonal matrix

$$
\left[\begin{array}{cc}
\frac{1}{\rho q} & 0 \\
0 & 1
\end{array}\right]
$$

the resulting system is symmetric hyperbolic. This matrix is the Hessian of the convex function

$$
P(q)+\frac{1}{2} \theta^{2} \quad \text { with } \quad P^{\prime \prime}(q)=\frac{1}{\rho(q) q} .
$$

Using the results of Friedrichs and Lax, [10], we can then show that system (2.18) admits another conservation law:

$$
\begin{aligned}
\frac{\partial}{\partial t} V(q, \theta) & +\frac{\partial}{\partial x}\left(q(\theta \sin \theta+\cos \theta)-\left(\int_{\bar{q}}^{q} \frac{1}{\rho(s) s} d s\right) \rho q \cos \theta\right) \\
& +\frac{\partial}{\partial y}\left(q(-\theta \cos \theta+\sin \theta)-\left(\int_{\bar{q}}^{q} \frac{1}{\rho(s) s} d s\right) \rho q \sin \theta\right)=0
\end{aligned}
$$

Here $0<\bar{q}<q_{\max }$ is an arbitrary constant, and

$$
V(q, \theta)=\frac{1}{2} \theta^{2}+\int_{\bar{q}}^{q} \frac{\rho(\mu) \mu d \mu}{a^{2}(\mu)} \int_{\bar{q}}^{\mu} \frac{1}{\rho(s) s} d s .
$$

To apply the above theory, we need $V$ to be convex as a function of $-\rho$ and $\theta$. This requires the inequality

$$
\begin{aligned}
0 & <V_{\rho \rho}=\frac{a^{2}}{\rho q} \frac{\partial}{\partial q}\left(\frac{a^{2}}{\rho q} \frac{\partial}{\partial q}\left[\int_{\bar{q}}^{q} \frac{\rho(\mu) \mu}{a^{2}(\mu)} \int_{\bar{q}}^{\mu} \frac{d s}{\rho(s) s}\right]\right) \\
& =\frac{a^{2}}{\rho q} \frac{\partial}{\partial q} \int_{\bar{q}}^{q} \frac{d s}{\rho(s) s}=\frac{a^{2}}{\rho^{2} q^{2}},
\end{aligned}
$$

which is always valid. 
Next, we consider plane wave solutions to our system (2.18), of the form (for fixed $\varphi$ between $-\pi$ and $\pi$ )

$$
w=f(x \cos \varphi+y \sin \varphi-s t) .
$$

The resulting matrix

$$
E^{-1}(A \cos \varphi+B \sin \varphi)
$$

has distinct eigenvalues

$$
\lambda_{\mp}=\left[\cos (\theta-\varphi)\left(1-\frac{1}{2 M^{2}}\right) \mp \sqrt{\frac{\cos ^{2}(\theta-\varphi)}{4 M^{4}}+\frac{\sin ^{2}(\theta-\varphi)}{M^{2}}}\right] .
$$

Suppose we have a steady solution to (2.18) having a discontinuity at $x \cos \varphi+$ $y \sin \varphi=0$.

Denote by $\left(q_{L}, \theta_{L}\right),\left(q_{R}, \theta_{R}\right)$, the left and right constant states and let

$$
\tilde{u}_{L}=q_{L} \cos \left(\theta_{L}-\varphi\right), \quad \tilde{v}_{L}=q_{L} \sin \left(\theta_{L}-\varphi\right),
$$

and similarly for $\tilde{u}_{R}, \tilde{v}_{R}$.

The jump conditions become

$$
\tilde{v}_{L}=\tilde{v}_{R}=\tilde{v}, \quad \rho\left(\tilde{u}_{L}, \tilde{v}\right) \tilde{u}_{L}=\rho\left(\tilde{u}_{R}, \tilde{v}\right) \tilde{u}_{R} .
$$

We are seeking steady, weak, shock solutions, locally of the form (2.26), so we consider points where an eigenvalue $\lambda_{-}$or $\lambda_{+}$vanishes. At such points, since

$$
\lambda_{+} \lambda_{-}=a^{2}\left(M^{2} \cos ^{2}(\theta-\varphi)-1\right),
$$

we must have

$$
M=\frac{1}{|\cos (\theta-\varphi)|} \geqslant 1 \text {. }
$$

We must check genuine nonlinearity of the field corresponding to this vanishing eigenvalue, $\lambda$.

The quadratic equation satisfied by each root is

$$
\lambda^{2} \frac{M^{2}}{q}+\lambda\left[\left(1-2 M^{2}\right) \cos (\theta-\varphi)\right]+q\left(M^{2} \cos ^{2}(\theta-\varphi)-1\right)=0 .
$$

Differentiating with respect to $q$ and $\theta$ at this point yields

$$
\begin{gathered}
\lambda_{q}=\frac{q \cos (\theta-\varphi) \partial M^{2} / \partial q}{\left(2 M^{2}-1\right)}=\frac{\cos (\theta-\varphi)\left[2 M^{2}+(\gamma-1) M^{4}\right]}{\left(2 M^{2}-1\right)}, \\
\lambda_{\theta}=\frac{2 q M^{2} \sin (\theta-\varphi)}{1-2 M^{2}} .
\end{gathered}
$$

We must check to be sure that $\left[\lambda_{q}, \lambda_{\theta}\right]^{T}=l^{T}$ is not orthogonal to the null eigenvector of

$$
-E^{-1}[A \cos \theta+B \sin \varphi]=\left[\begin{array}{cc}
\frac{a^{2}}{q}\left(1-\dot{M}^{2}\right) \cos (\theta-\varphi) & -a^{2} \sin (\theta-\varphi) \\
-\sin (\theta-\varphi) & -q \cos (\theta-\varphi)
\end{array}\right]
$$

at this point. 
We may take this eigenvector to be

$$
[q \cos (\theta-\varphi),-\sin (\theta-\varphi)]^{T}=r^{T} .
$$

A simple calculation yields

$$
l^{T} r=q \gamma M^{2} /\left(2 M^{2}-1\right) \neq 0
$$

Thus, the Lax $k$-shock condition is valid here. This means that (2.28) is valid, and that $\lambda\left(\tilde{u}_{L}, \tilde{v}\right)>0>\lambda\left(\tilde{u}_{R}, \tilde{v}\right)$.

If $\tilde{u}_{L}, \tilde{u}_{R}>0$, then it is $\lambda_{-}$which is the relevant eigenvalue, and we have from (2.27), (2.29):

$$
\tilde{u}_{L}^{2} / a_{L}^{2}>1
$$

Thus, we have, in this case,

(a) $\tilde{u}_{L} / a_{L}>1$

Since

$$
\frac{\partial}{\partial u} \rho(u, \tilde{v}) u=-\rho\left(1-M^{2}\right)
$$

changes sign for positive $u$ iff $u=a$, there exists exactly one positive root $\tilde{u}_{R}$ of (2.28) and it satisfies

(b) $0<\tilde{u}_{R} / a_{R}<1$.

The criteria (2.30) (a), (b) are the usual physically correct ones for shocks satisfying (2.1) $-(2.4)$.

If $\tilde{u}_{L}, \tilde{u}_{R}<0$, then it is $\lambda_{+}$which is relevant, and we have from (2.27), (2.29):

$$
\tilde{u}_{R}^{2} / a_{R}^{2}>1 \text {. }
$$

We arrive at, as above,

(a) $0<-\tilde{u}_{L} / a_{L}<1$,

(b) $-\tilde{u}_{R} / a_{R}>1$,

which is, again, the usual physical restriction.

We have thus proven

THEOREM 2.1. Solutions of (2.1)-(2.4), having weak shocks, satisfy the inequality

$$
\begin{aligned}
\frac{\partial}{\partial x}(q(\theta \sin \theta+ & \left.\cos \theta)-\left(\int_{\bar{q}}^{q} \frac{1}{\rho(s) s} d s\right) \rho q \cos \theta\right) \\
& +\frac{\partial}{\partial y}\left(q(-\theta \cos \theta+\sin \theta)-\left(\int_{\bar{q}}^{q} \frac{1}{\rho(s) s} d s\right) \rho q \sin \theta\right) \leqslant 0,
\end{aligned}
$$

iff the shocks are physically correct in the sense of (2.30), (2.31).

III. A Class of Entropy Condition Satisfying Approximations. Now we shall construct difference approximations to (2.1), (2.4) whose limit solutions satisfy inequality (2.32). Thus, these limits have only physically correct shocks. A wide class of these schemes will be constructed. They can be programmed using only simple (and simplifying) changes from existing codes which use density biasing. Ours will use flux biasing. In addition to satisfying the entropy conditions, the schemes will also have monotone, sharp, discrete shocks, as explained in Section IV below. 
We set up a grid

$$
x_{j}=j \Delta x, \quad y_{k}=k \Delta y, \quad j, k=0, \pm 1, \pm 2, \ldots,
$$

and seek a grid function

$$
\Phi_{j k} \approx \Phi(j \Delta x, k \Delta y) .
$$

Let

$$
\overleftarrow{\delta}_{x} \Phi=\frac{\Phi_{j k}-\Phi_{j-1, k}}{\Delta x}, \quad \vec{\delta}_{x} \Phi=\frac{\Phi_{j+1, k}-\Phi_{j k}}{\Delta x}
$$

define the backward and forward difference operators, with $\overleftarrow{\delta}_{y}, \vec{\delta}_{y}$, defined analogously.

We also define the operations

$$
A^{+}=\max (A, 0), \quad A^{-}=\min (A, 0) .
$$

On the grid, we let the absolute speed be defined through

$$
q=\sqrt{\left(\left(\overleftarrow{\delta}_{x} \Phi\right)^{+}-\left(\vec{\delta}_{x} \Phi\right)^{-}\right)^{2}+\left(\left(\overleftarrow{\delta}_{y} \Phi\right)^{+}-\left(\vec{\delta}_{y} \Phi\right)^{-}\right)^{2}}
$$

We then use (3.2) to define $\rho, a$, and $M$ on the grid.

Next we consider a numerical flux which is a Lipschitz continuous function of two variables $h\left(q_{1}, q_{0}\right)$ which is consistent with the function $-\rho q$, i.e.,

$$
h(q, q) \equiv-\rho(q) q
$$

and which satisfies the inequality

$$
\operatorname{sgn}\left(q_{1}-q_{0}\right)\left[h\left(q_{1}, q_{0}\right)+\rho q\right] \leqslant 0
$$

for all $q$ between $\left(q_{0}, q_{1}\right)$.

Such numerical fluxes are building blocks for "E" schemes, and were introduced by the first author in [23]. They include monotone schemes as special cases. In our context, $h\left(q_{1}, q_{0}\right)$ corresponds to a monotone scheme if it is nonincreasing in its first argument, nondecreasing in its second. In the next section we give examples of these concepts, together with comparisons to density biasing algorithms. If $h$ is a monotone flux, then for $q$ between $q_{0}$ and $q_{1}$,

$$
\begin{aligned}
\operatorname{sgn}\left(q_{1}-\right. & \left.q_{0}\right)\left[h\left(q_{1}, q_{0}\right)+\rho(q) q\right] \\
= & \operatorname{sgn}\left(q_{1}-q\right)\left[h\left(q_{1}, q_{0}\right)-h\left(q, q_{0}\right)\right] \\
& +\operatorname{sgn}\left(q-q_{0}\right)\left[h\left(q, q_{0}\right)-h(q, q)\right] \leqslant 0 ;
\end{aligned}
$$

hence $h$ is an E flux.

For any such flux, our algorithm approximating $(2.1)-(2.4)$ is

$$
\begin{aligned}
0= & \vec{\delta}_{x}\left[\frac{\left(\overleftarrow{\delta}_{x} \Phi\right)^{+}}{q}\left[-h\left(q_{j k}, q_{j-1, k}\right)\right]\right]+\overleftarrow{\delta}_{x}\left[\frac{\left(\vec{\delta}_{x} \Phi\right)^{-}}{q}\left[-h\left(q_{j k}, q_{j+1, k}\right)\right]\right] \\
& +\vec{\delta}_{y}\left[\frac{\left(\overleftarrow{\delta}_{y} \Phi\right)^{+}}{q}\left[-h\left(q_{j k}, q_{j, k-1}\right)\right]\right]+\overleftarrow{\delta}_{y}\left[\frac{\left(\vec{\delta}_{y} \Phi\right)^{-}}{q}\left[-h\left(q_{j k}, q_{j, k+1}\right)\right]\right]
\end{aligned}
$$

We need two minor technical assumptions, before stating our main theorem. 
Assumption (3.1). As $\Delta x, \Delta y \rightarrow 0, \bar{\delta}_{x} \Phi$ is always of one sign in a given region $\Omega \subset R^{2}$, and $\bar{\delta}_{y} \Phi$ is always of one (perhaps different) sign in this same region.

Assumption (3.2). The oscillation

$$
\sup \left|\Delta x \overleftarrow{\delta}_{x} \vec{\delta}_{x} \Phi\right|+\sup \left|\Delta y \overleftarrow{\delta}_{y} \vec{\delta}_{y} \Phi\right|+\sup (\Delta x+\Delta y)\left(\left|\overleftarrow{\delta}_{x} \vec{\delta}_{y} \Phi\right|\right)
$$

is sufficiently small, as $\Delta x \rightarrow 0, \Delta y \rightarrow 0$ in $\Omega$ (i.e., discrete shocks are weak).

Given these two assumptions, we can now state

THEOREM 3.1. Suppose $\left\{\Phi_{j k}\right\}$ is a solution to (3.6), and $\Phi_{j k}, \overleftarrow{\delta}_{x} \Phi_{j k}$, and $\overleftarrow{\delta}_{y} \Phi_{j k}$ converge boundedly a.e. in $\Omega$ to $\Phi, \Phi_{x}$, and $\Phi_{y}$, respectively. Then, in $\Omega, \Phi$ is a weak solution of (2.1)-(2.4), which satisfies the entropy inequality (2.32).

IV. Examples Based on "E" Approximations. In this section we shall give examples of schemes of the type (3.6), where the function $h$ is an E flux. We shall compare these flux biasing algorithms, with the density biasing methods of [6], [14], and [17].

Example (1): The Engquist-Osher scheme (E-O). In order to construct this algorithm here, we note that

$$
\begin{aligned}
\frac{d}{d q}(-\rho q) & =-\rho\left(1-\frac{q^{2}}{a^{2}}\right)>0 \quad \text { iff } M>1 \\
& =-\rho\left(1-\frac{q^{2}}{a^{2}}\right)<0 \quad \text { iff } M<1 .
\end{aligned}
$$

However,

$$
M>1 \Leftrightarrow q>q^{*}=a^{*}=\sqrt{\frac{M_{\infty}^{-2}+(\gamma-1) / 2}{(\gamma+1) / 2}}
$$

and

$$
\rho\left(q^{*}\right)=\rho^{*}=\left(1-\frac{\gamma-1}{2} M_{\infty}^{2}\left(\left(a^{*}\right)^{2}-1\right)\right)^{1 /(\gamma-1)} .
$$

We define the function

$$
\begin{array}{ll}
(\rho q)_{-} \equiv 0 & \text { if } q \leqslant q^{*} \Leftrightarrow M \leqslant 1, \\
(\rho q)_{-} \equiv \rho q-\rho^{*} q^{*} & \text { if } q>q^{*} \Leftrightarrow M>1 .
\end{array}
$$

Finally, we set

$$
-h^{\mathrm{EO}}\left(q_{j k}, q_{j-1, k}\right)=\rho_{j k} q_{j k}-\Delta x \bar{\delta}_{x}\left(\rho_{j k} q_{j k}\right)_{-} .
$$

It is easy to see that this flux is monotone. In this case our scheme becomes

$$
\begin{aligned}
0= & \vec{\delta}_{x}\left[\frac{\left(\overleftarrow{\delta}_{x} \Phi\right)^{+}}{q}\left[\rho q-\Delta x \overleftarrow{\delta}_{x}(\rho q)_{-}\right]\right]+\overleftarrow{\delta}_{x}\left[\frac{\left(\vec{\delta}_{x} \Phi\right)^{-}}{q}\left[\rho q+\Delta x \vec{\delta}_{x}(\rho q)_{-}\right]\right] \\
& +\vec{\delta}_{y}\left[\frac{\left(\overleftarrow{\delta}_{y} \Phi\right)^{+}}{q}\left[\rho q-\Delta y \overleftarrow{\delta}_{y}(\rho q)_{-}\right]\right]+\overleftarrow{\delta}_{y}\left[\frac{\left(\vec{\delta}_{y} \Phi\right)^{-}}{q}\left[\rho q+\Delta y \vec{\delta}_{y}(\rho q)_{-}\right]\right]
\end{aligned}
$$

In the subsonic case, this scheme is merely conventional central differencing (and is second-order accurate, modulo a simple change in the definition of $\rho$ ). However, we bias the mass flux $\rho q$, rather than $\rho$, to achieve our upwinding. We also obtain a simpler algorithm in the supersonic region, as will be shown below. 
Example (2): Godunov's scheme [11]. The precise definition, [23], is

$$
\begin{aligned}
& -h^{\mathrm{G}}\left(q_{j k}, q_{j-1, k}\right)=\max _{q_{j-1, k} \leqslant q \leqslant q_{j k}} \rho(q) q \text { if } q_{j-1, k}<q_{j k}, \\
& -h^{\mathrm{G}}\left(q_{j k}, q_{j-1, k}\right)=\min _{q_{j-1, k} \geqslant q \geqslant q_{j k}} \rho(q) q \text { if } q_{j-1, k}>q_{j k} .
\end{aligned}
$$

It is enlightening to discuss the physics behind this. The flux is constructed by solving the Riemann initial value problem

$$
-\rho_{t}-(\rho q)_{x}=0
$$

for $t>0$, with initial data

$$
\begin{array}{ll}
q(x, 0) \equiv q_{j-1, k} & \text { if } x<0, \\
q(x, 0) \equiv q_{j k} & \text { if } x \geqslant 0 .
\end{array}
$$

One obtains the unique, physically correct (entropy condition satisfying) solution, which is of the form $q(x / t)$. One then evaluates $\rho(q) q$ at $x / t=0$.

Then

$$
-h^{\mathrm{G}}\left(q_{j k}, q_{j-1, k}\right)=\rho(q(0)) q(0) .
$$

Now

$$
\frac{d^{2}}{d(-\rho)^{2}}(-\rho(q) q)=\frac{q}{\rho M^{2}}\left(\gamma+\frac{1}{M^{2}}\right)>0 .
$$

Thus, $-\rho q$ is convex as a function of $-\rho$.

This yields a simplification of (4.5):

$$
h^{\mathrm{G}}\left(q_{j k}, q_{j-1, k}\right)=h^{\mathrm{EO}}\left(q_{j k}, q_{j-1, k}\right)
$$

unless

$$
q_{j-1, k}>q^{*}>q_{j k}
$$

We then compute the shock speed

$$
s_{j-1 / 2, k}=\frac{\rho_{j k} q_{j k}-\rho_{j-1, k} q_{j-1, k}}{\rho_{j k}-\rho_{j-1, k}}
$$

and define, in this case,

$$
\begin{aligned}
& -h^{\mathrm{G}}\left(q_{j k}, q_{j-1, k}\right)=\frac{\rho_{j k} q_{j k}+\rho_{j-1, k}, q_{j-1, k}}{2}+\frac{1}{2}\left|s_{j-1 / 2, k}\right|\left(\rho_{j k}-\rho_{j-1, k}\right) \\
& =\rho_{j-1, k} q_{j-1, k} \text { if } s_{j-1 / 2, k}>0, \\
& =\rho_{j k} q_{j k} \quad \text { if } s_{j+1 / 2, k}<0 .
\end{aligned}
$$

Inserting this into (3.6) gives us the corresponding approximation to the full potential equation.

An equivalent formulation giving us Godunov's flux in all cases is

$$
-h^{\mathrm{G}}\left(q_{j k}, q_{j-1, k}\right)=\text { the expression in (4.12), }
$$

unless $q_{j-1, k}<q^{*}<q_{j k}$, in which case

$$
-h^{\mathrm{G}}\left(q_{j k}, q_{j-1, k}\right)=\rho^{*} q^{*} .
$$


Godunov's is the ultimate E scheme (see [23]). However, the Engquist-Osher scheme is simpler to program, and has $C^{1}$ flux functions, while Godunov's flux has a jump discontinuity in derivative at sonic shock points.

Formula (4.12) is precisely the algorithm for Murman's conservative upwind scheme, devised in [21], for the small disturbance equation, with $-\rho q$ replaced by $(\gamma+1) \rho^{2} / 2+k \rho, k$ a positive constant. This method does not yield an E scheme, and admits stable expansion shocks. Transonic small-disturbance calculations using E-O, [13] and later Godunov's method [12], were found to be much more robust and reliable than those using Murman's algorithm.

Many other fixes for Murman's algorithm have been recently devised, e.g., [16], [29]. They are typically of this type: Define $h$ as in (4.12) unless $q_{j-1, k}<q^{*}<q_{j k}$, i.e., at a sonic rarefaction. The flux will generate an $\mathrm{E}$ scheme if one defines, in this case, $h$ to satisfy

$$
h\left(q_{j k}, q_{j-1, k}\right) \leqslant-\rho^{*} q^{*}=h^{\mathrm{G}}\left(q_{j k}, q_{j-1, k}\right) .
$$

Many other possible E schemes exist. However, each method we have described here has the following properties:

(a) The scheme is fully one-sided, i.e.,

$$
\begin{aligned}
h\left(q_{j k}, q_{j-1, k}\right) & \equiv-\rho_{j k} q_{j k} & & \text { if } q_{j k}, q_{j-1, k}<q^{*}, \\
& \equiv-\rho_{j-1, k} q_{j-1, k} & & \text { if } q_{j k}, q_{j-1, k}>q^{*} .
\end{aligned}
$$

(b) One-dimensional steady discrete shocks are exact except for one transition point for any of the above-mentioned E schemes, except for E-O, which has two transition points. The extra point follows because of E.O.'s smoother flux function (see [9]).

This means if $\Phi(x, y) \equiv \Phi(x)$, with, say $u=\varphi_{x}>0$, defining a steady shock

$$
\begin{array}{ll}
u \equiv u^{L} & \text { for } x<0, \\
u \equiv u^{R} & \text { for } x>0,
\end{array}
$$

with $M^{L}>1>M^{R}$, then for all the above methods except E.O., we have as discrete solutions to (3.6):

$$
\begin{array}{ll}
u_{j} \equiv u^{L}, & j<0, \\
u_{j} & \equiv u^{R}, \quad j>0,
\end{array} u^{L} \geqslant u_{0} \geqslant u^{R}, u_{0} \text { otherwise arbitrary }
$$

For E.O., we have

$$
\begin{array}{ll}
u_{j} \equiv u^{L}, & j<0, \\
u_{j} \equiv u^{R}, & j>1,
\end{array} \quad u^{L} \geqslant u_{0} \geqslant q^{*}>u_{1}>u^{R},
$$

with $\rho_{0} u_{0}+\rho_{1} u_{1} \equiv \rho_{L} u_{L}+\rho^{*} q^{*}$. See [9] for analysis of this, and for some results in two dimensions. We also conjecture here that two-dimensional supersonic-subsonic discrete shocks are monotone, and exact, except for a finite number of grid points, for any of the FP algorithms mentioned above.

Thus, we have the desirable properties of programming simplicity, sharp discrete shocks, dissipation of expansion shocks, and, with E.O., smoothness of flux functions. This last property helps in accelerating iterative methods to convergence [15]. 
Density biasing algorithms for E.O. have been in use for some time, e.g., [6], [14], [17]. We now compare the $x$ differencing in the algorithms of [6], [14], [17], with that of (4.4). Using E.O., we have (if $\overleftarrow{\delta}_{x} \Phi>0$ for all relevant points)

Density Biasing.

$$
\overleftarrow{\delta}_{x}\left[\bar{\rho}_{j+1, k} \vec{\delta}_{x} \Phi\right] \approx \frac{\partial}{\partial x} \rho u
$$

with

$$
\bar{\rho}_{j+1, k}=\rho_{j+1, k}-\nu_{j k}\left(\rho_{j+1, k}-\rho_{j k}\right), \quad \nu_{j k}=\max \left[0,1-\frac{1}{\tilde{M}_{j k}^{2}}\right]
$$

for $\tilde{M}_{j k}$ some representation of the local Mach number.

E.O. Flux Biasing.

$$
\overleftarrow{\delta}_{x}\left[\overline{\bar{\rho}}_{j+1, k} \vec{\delta}_{x} \Phi\right]
$$

with

$$
\overline{\bar{\rho}}_{j+1, k}=\rho_{j+1, k}-\frac{\left[\left(\rho_{j+1, k} q_{j+1, k}\right)_{-}-\left(\rho_{j k} q_{j k}\right)_{-}\right]}{q_{j+1, k}} .
$$

Taylor's theorem shows the equivalence of $\bar{\rho}$ and $\overline{\bar{\rho}}$ up to terms of order $\left(\Delta x \vec{\delta}_{x} q_{j k}\right)^{2}$. If centered differences are used in the definition of $u$ and $v$ in $q_{j k}$, they both yield the identical subsonic second-order accurate differencing. In supersonic regions, the algorithms are quite different.

Density Biasing.

$$
\overleftarrow{\delta}_{x}\left(\left[\frac{\rho_{j+1, k}}{\tilde{M}_{j k}^{2}}+\left(1-\frac{1}{\tilde{M}_{j k}^{2}}\right) \rho_{j k}\right] \vec{\delta}_{x} \Phi\right)
$$

E.O. Flux Biasing.

$$
\bar{\delta}_{x}\left(\left(\frac{\rho_{j k} q_{j k}}{q_{j+1, k}}\right) \vec{\delta}_{x} \Phi\right) .
$$

We again emphasize that our simpler formula differs from the density biasing one as follows:

$$
\frac{\rho_{j+1, k}}{\tilde{M}_{j k}^{2}}+\left(1-\frac{1}{\tilde{M}_{j k}^{2}}\right) \rho_{j k}=\frac{\rho_{j k} q_{j k}}{q_{j+1, k}}+O(\Delta x)^{2} .
$$

It is perhaps amusing to show this in detail. Dropping the $j, k$ and $\sim$ dependence, we have

$$
\begin{aligned}
\rho+\frac{1}{M^{2}} \Delta x \vec{\delta}_{x} \rho & =\rho+\frac{1}{M^{2}}\left(\frac{-\rho q}{c^{2}}\right) \Delta x q_{x}+O(\Delta x)^{2} \\
& =\rho-\frac{\rho}{q} \Delta x q_{x}+O(\Delta x)^{2}
\end{aligned}
$$

while

$$
\frac{p q}{q(x+\Delta x)}=\rho-\frac{\rho \Delta x \vec{\delta}_{x} q}{q(x+\Delta x)}=\rho-\frac{\rho}{q} \Delta x q_{x}+O(\Delta x)^{2} .
$$

Thus, the two expressions agree up to $O(\Delta x)^{2}$. 
V. Proof of Main Theorem. We shall prove a discrete version of inequality (2.32). Let the four terms in (3.6) be defined by

$$
[\mathrm{I}]+[\mathrm{II}]+[\mathrm{III}]+[\mathrm{IV}]
$$

By Assumption (3.1), one of the first two, and one of the last two, terms is zero.

Define, in the nonvanishing terms,

$$
\begin{aligned}
\cos \theta^{\mathrm{I}}=\frac{\overleftarrow{\delta}_{x} \Phi}{q}, & \sin \theta^{\mathrm{III}}=\frac{\overleftarrow{\delta}_{y} \Phi}{q} \\
\cos \theta^{\mathrm{II}}=\frac{\vec{\delta}_{x} \Phi}{q}, & \sin \theta^{\mathrm{IV}}=\frac{\vec{\delta}_{y} \Phi}{q} .
\end{aligned}
$$

In all cases, we can uniquely define the relevant $\theta^{\mathrm{I}}, \theta^{\mathrm{II}}, \theta^{\mathrm{III}}$, or $\theta^{\mathrm{IV}}$. For example, if the region $\Omega$ is such that

$$
\overleftarrow{\delta}_{x} \Phi>0>\vec{\delta}_{y} \Phi
$$

then

$$
\theta^{\mathrm{I}}=\theta^{\mathrm{IV}}=\cos ^{-1}\left(\frac{\overleftarrow{\delta}_{x} \Phi}{q}\right)=\sin ^{-1}\left(\frac{\vec{\delta}_{y} \Phi}{q}\right)
$$

where, in this case,

$$
q=\sqrt{\left(\overleftarrow{\delta}_{x} \Phi\right)^{2}+\left(\vec{\delta}_{y} \Phi\right)^{2}}
$$

In general, we let

$$
\chi(\mu) \equiv 1 \quad \text { if } \mu \geqslant 0, \quad \chi(\mu) \equiv 0 \quad \text { if } \mu<0 .
$$

We shall derive the following discrete inequality:

$$
\begin{aligned}
& \vec{\delta}_{x}\left[\overleftarrow{\delta}_{x} \Phi+\right.\left(\int_{\bar{q}}^{q} \frac{1}{\rho(s) s} d s\right) \frac{\left(\overleftarrow{\delta}_{x} \Phi\right)^{+}}{q} h\left(q_{j k}, q_{j-1, k}\right) \\
&+\left(\int_{\bar{q}}^{q_{j-1, k}} \frac{1}{\rho(s) s} d s\right) \frac{\left(\overleftarrow{\delta}_{x} \Phi\right)^{-}}{q_{j-1, k}} h\left(q_{j-1, k} q_{j k}\right) \\
&+ \chi\left(\cos \theta^{\mathrm{I}}\right)\left(q_{j-1, k} \sin \theta_{j-1, k}^{\mathrm{I}}\right) \theta^{\mathrm{I}} \\
&\left.+\left(1-\chi\left(\cos \theta^{\mathrm{II}}\right)\right)\left(q_{j k} \sin \theta_{j k}^{\mathrm{II}}\right) \theta_{j-1, k}^{\mathrm{II}}\right] \\
&+ \vec{\delta}_{y}\left[\overleftarrow{\delta}_{y} \Phi+\left(\int_{\bar{q}}^{q} \frac{1}{\rho(s) s} d s\right) \frac{\left(\overleftarrow{\delta}_{y} \Phi\right)^{+}}{q} h\left(q_{j k}, q_{j, k-1}\right)\right. \\
&+\left(\int_{\bar{q}}^{q_{j, k-1}} \frac{1}{\rho(s) s} d s\right) \frac{\left(\overleftarrow{\delta}_{y} \Phi\right)^{-}}{q_{j, k-1}} h\left(q_{j, k-1}, q_{j k}\right) \\
&-\chi\left(\sin \theta^{\mathrm{III}}\right)\left(q_{j, k-1} \cos \theta_{j, k-1}^{\mathrm{III}}\right) \theta^{\mathrm{III}} \\
&\left.-\left(1-\chi\left(\sin \theta^{\mathrm{IV}}\right)\right)\left(q_{j k} \cos \theta_{j k}^{\mathrm{IV}}\right) \theta_{j, k-1}^{\mathrm{IV}}\right] \leqslant 0 .
\end{aligned}
$$

The Lebesgue dominated convergence theorem will then give us (2.32). Moreover, the Lax-Wendroff theorem [30] implies that the limit is a weak solution of (2.1)-(2.4).

We prove (5.3) by multiplying (3.6) by $\int_{\frac{q}{q}}(1 / \rho(s) s) d s$ and adding it to the expression on the left in (3.3). 
We first consider

$$
\begin{aligned}
\int_{\bar{q}}^{q} \frac{1}{\rho(s) s} d s \vec{\delta}_{x}\left[\frac{\left(\overleftarrow{\delta}_{x} \Phi\right)^{+}}{q}\left(-h\left(q, q_{j-1, k}\right)\right)\right] \\
+\vec{\delta}_{x}\left[q \frac{\left(\overleftarrow{\delta}_{x} \Phi\right)^{+}}{q}+\int_{\bar{q}}^{q} \frac{1}{\rho(s) s} d s \frac{\left(\overleftarrow{\delta}_{x} \Phi\right)^{+}}{q} h\left(q, q_{j-1, k}\right)\right] \\
=\frac{\left(\vec{\delta}_{x} \Phi\right)^{+}}{q_{j+1, k}}\left(\int_{q}^{q_{j+1, k}} \frac{d s}{\rho(s) s}\right) \frac{h\left(q_{j+1, k}, q\right)}{\Delta x} \\
+\frac{\left(\vec{\delta}_{x} \Phi\right)^{+}}{q_{j+1, k}} \vec{\delta}_{x} q+q \vec{\delta}_{x}\left(\frac{\left(\overleftarrow{\delta}_{x} \Phi\right)^{+}}{q}\right) \\
=\frac{\left(\vec{\delta}_{x} \Phi\right)^{+}}{q_{j+1, k}}\left(\int_{q}^{q_{j+1, k}} \frac{d s}{\rho(s) s}\left(h\left(q_{j+1, k}, q\right)+\rho(s) s\right)\right)+q \vec{\delta}_{x}\left(\frac{\left(\overleftarrow{\delta}_{x} \Phi\right)^{+}}{q}\right) \\
\leqslant
\end{aligned}
$$

(since $h$ is an E flux).

Now we add

$$
\chi\left(\cos \theta^{\mathrm{I}}\right) \vec{\delta}_{x}\left(\left(q_{j-1, k} \sin \theta_{j-1, k}^{\mathrm{I}}\right) \theta^{\mathrm{I}}\right)
$$

to the above, arriving at

$$
\begin{aligned}
q \chi\left(\cos \theta^{\mathrm{I}}\right) & {\left[\vec{\delta}_{x} \cos \theta^{\mathrm{I}}+\left(\sin \theta^{\mathrm{I}}\right) \vec{\delta}_{x} \theta^{\mathrm{I}}\right]+\chi\left(\cos \theta^{\mathrm{I}}\right) \theta^{\mathrm{I}} \hat{\delta}_{x}\left[q \sin \theta^{\mathrm{I}}\right] } \\
= & q\left|\cos \theta^{\mathrm{I}}\right|\left(\frac{\cos \left(\Delta x \vec{\delta}_{x} \theta^{\mathrm{I}}\right)-1}{\Delta x}\right) \\
& +q \chi\left(\cos \theta^{\mathrm{I}}\right)\left(\sin \theta^{\mathrm{I}}\right)\left(\vec{\delta}_{x} \theta^{\mathrm{I}}-\frac{\sin \left(\Delta x \vec{\delta}_{x} \theta^{\mathrm{I}}\right)}{\Delta x}\right) \\
& +\chi\left(\cos \theta^{\mathrm{I}}\right) \theta^{\mathrm{I}} \hat{\delta}_{x}\left[q \sin \theta^{\mathrm{I}]}\right. \\
\leqslant & \chi\left(\cos \theta^{\mathrm{I}}\right) \theta^{\mathrm{I}} \hat{\delta}_{x}\left[q \sin \theta^{\mathrm{I}}\right] \text { if }\left|\Delta x \vec{\delta}_{x} \theta^{\mathrm{I}}\right| \text { is sufficiently small. }
\end{aligned}
$$

Next, we consider

$$
\begin{aligned}
\int_{\bar{q}}^{q} \frac{1}{\rho(s) s} d s \vec{\delta}_{y}\left[\frac{\left(\overleftarrow{\delta}_{y} \Phi\right)^{+}}{q}\left(-h\left(q_{j k}, q_{j, k-1}\right)\right)\right] \\
+\vec{\delta}_{y}\left[q \frac{\left(\overleftarrow{\delta}_{y} \Phi\right)^{+}}{q}+\int_{\bar{q}}^{q} \frac{1}{\rho(s) s} d s \frac{\left(\overleftarrow{\delta}_{y} \Phi\right)^{+}}{q} h\left(q_{j k}, q_{j, k-1}\right)\right] \\
=\frac{\left(\vec{\delta}_{y} \Phi\right)^{+}}{q_{j+1, k}} \int_{q_{j k}}^{q_{j k+1}} \frac{1}{\rho(s) s} \frac{h\left(q_{j, k+1}, q_{j k}\right)}{\Delta y} \\
\quad+\frac{\left(\vec{\delta}_{y} \Phi\right)^{+}}{q_{j+1, k}} \vec{\delta}_{y} q_{j k}+q_{j k} \vec{\delta}_{y}\left(\frac{\left(\overleftarrow{\delta}_{y} \Phi\right)^{+}}{q}\right) \\
\leqslant q_{j k} \chi\left(\sin \theta^{\mathrm{III}}\right) \vec{\delta}_{y}\left(\sin \theta^{\mathrm{III}}\right)
\end{aligned}
$$

We now add

(again using the fact that $h$ is an E flux).

$$
-\chi\left(\sin \theta^{\mathrm{III}}\right) \vec{\delta}_{y}\left(\left(q_{j, k-1} \cos \theta_{j, k-1}^{\mathrm{III}}\right) \theta^{\mathrm{III}}\right)
$$


to the above, arriving at

$$
\begin{aligned}
& q \chi\left(\sin \theta^{\mathrm{III}}\right)\left(\vec{\delta}_{y} \sin \theta^{\mathrm{III}}-\cos \theta^{\mathrm{III}} \vec{\delta}_{y} \theta^{\mathrm{III}}\right)-\chi\left(\sin \theta^{\mathrm{III}}\right) \theta^{\mathrm{III}} \vec{\delta}_{y}\left(q \cos \theta^{\mathrm{III}}\right) \\
&= q\left|\sin \left(\theta^{\mathrm{III}}\right)\right|\left(\frac{\cos \left(\Delta y \vec{\delta}_{y} \theta^{\mathrm{III}}\right)}{\Delta y}-1\right)+q\left(\chi \sin \theta^{\mathrm{III}}\right) \\
& \times \cos \left(\theta^{\mathrm{III}}\right)\left(\frac{\sin \left(\Delta y \vec{\delta}_{y} \theta^{\mathrm{III}}\right)}{\Delta y}-\vec{\delta}_{y} \theta^{\mathrm{III}}\right) \\
&-\chi\left(\sin \theta^{\mathrm{III}}\right) \theta^{\mathrm{III}} \widehat{\delta}_{y}\left(q \cos \theta^{\mathrm{III}}\right) \\
& \leqslant-\chi\left(\sin \theta^{\mathrm{III}}\right) \theta^{\mathrm{III}} \widehat{\delta}_{y}\left(q \cos \theta^{\mathrm{III}}\right) \text { if }\left|\Delta y \vec{\delta}_{y} \theta^{\mathrm{III}}\right| \text { is sufficiently small. }
\end{aligned}
$$

Third, we consider

$$
\begin{aligned}
\int_{\bar{q}}^{q} \frac{1}{\rho(s) s} d s \overleftarrow{\delta}_{x}\left(\frac{\left(\vec{\delta}_{x} \Phi\right)^{-}}{q_{j k}}\left(-h\left(q_{j k}, q_{j+1, k}\right)\right)\right) \\
+\bar{\delta}_{x}\left[\int_{\bar{q}}^{q} \frac{1}{\rho(s) s} d s \frac{\left(\vec{\delta}_{x} \Phi\right)^{-}}{q_{j k}} h\left(q_{j k}, q_{j+1, k}\right)+q_{j k} \frac{\left(\vec{\delta}_{x} \Phi\right)^{-}}{q_{j k}}\right] \\
=\frac{1}{\Delta x} \int_{q_{j-1, k}}^{q_{j k}}\left(\overleftarrow{\delta}_{x} \Phi\right)^{-} h\left(q_{j-1, k}, q_{j k}\right) \frac{d s}{\rho(s) s} \frac{1}{q_{j-1, k}} \\
+\frac{\left(\overleftarrow{\delta}_{x} \Phi\right)^{-}}{q_{j-1, k}} \overleftarrow{\delta}_{x} q+q_{j k} \overleftarrow{\delta}_{x}\left(\frac{\left(\vec{\delta}_{x} \Phi\right)^{-}}{q_{j k}}\right) \\
=\frac{1}{\Delta x} \int_{q_{j-1, k}}^{q_{j k}} \frac{\left(\overleftarrow{\delta}_{x} \Phi\right)^{-}}{q_{j-1, k}} \frac{1}{\rho(s) s} d s\left(h\left(q_{j-1, k}, q_{j k}\right)+\rho(s) s\right) \\
\quad+q_{j k} \overleftarrow{\delta}_{x}\left(\frac{\left(\vec{\delta}_{x} \Phi\right)^{-}}{q_{j k}}\right) \\
\leqslant q_{j k}\left(1-\chi\left(\cos \theta^{\mathrm{II}}\right)\right) \overleftarrow{\delta}_{x}\left(\cos \theta^{\mathrm{II}}\right)
\end{aligned}
$$

(using the fact that $h$ is an E flux).

We now add

$$
\left(1-\chi\left(\cos \theta^{\mathrm{II}}\right)\right) \vec{\delta}_{x}\left(q \sin \theta^{\mathrm{II}} \theta_{j-1, k}^{\mathrm{II}}\right)
$$

to $(5.8)$, arriving at

$$
\begin{aligned}
(1-\chi & \left.\left(\cos \theta^{\mathrm{II}}\right)\right) q\left[\overleftarrow{\delta}_{x}\left(\cos \theta^{\mathrm{II}}\right)+\left(\sin \theta^{\mathrm{II}} \bar{\delta}_{x} \theta^{\mathrm{II}}\right)\right] \\
& +\left(1-\chi\left(\cos \theta^{\mathrm{II}}\right)\right) \theta^{\mathrm{II}}\left(\vec{\delta}_{x}\left(q \sin \theta^{\mathrm{II}}\right)\right) \\
= & -q\left|\cos \theta^{\mathrm{II}}\right| \frac{\left(1-\cos \left(\Delta x \bar{\delta}_{x} \theta^{\mathrm{II}}\right)\right)}{\Delta x} \\
& +\left(1-\chi\left(\cos \theta^{\mathrm{II}}\right)\right)\left(\sin \theta^{\mathrm{II}}\right)\left(\bar{\delta}_{x} \theta^{\mathrm{II}}-\frac{\sin \left(\Delta x \bar{\delta}_{x} \theta^{\mathrm{II}}\right)}{\Delta x}\right) \\
& +\left(1-\chi\left(\cos \theta^{\mathrm{II}}\right)\right) \theta^{\mathrm{II}} \vec{\delta}_{x}\left(q \sin \theta^{\mathrm{II}}\right) \\
\leqslant & \left(1-\chi\left(\cos \theta^{\mathrm{II}}\right)\right) \theta^{\mathrm{II}} \vec{\delta}_{x}\left(q \sin \theta^{\mathrm{II}}\right) \text { if }\left|\Delta x \delta_{x} \theta^{\mathrm{II}}\right| \text { is small enough. }
\end{aligned}
$$


Finally, we consider

$$
\begin{aligned}
& \int_{\bar{q}}^{q} \frac{1}{\rho(s) s} d s \bar{\delta}_{y}\left[\frac{\left(\vec{\delta}_{y} \Phi\right)^{-}}{q}\left[-h\left(q_{j k}, q_{j, k+1}\right)\right]\right] \\
& \quad+\bar{\delta}_{y}\left[\int_{\bar{q}}^{q_{j k}} \frac{1}{\rho(s) s} d s \frac{\left(\vec{\delta}_{y} \Phi\right)^{-}}{q_{j k}} h\left(q_{j k}, q_{j, k+1}\right)+q_{j k} \frac{\left(\vec{\delta}_{y} \Phi\right)^{-}}{q_{j k}}\right] \\
& \quad=\frac{1}{\Delta y} \int_{q_{j, k-1}}^{q_{j k}} \frac{1}{\rho(s) s} d s \frac{\left(\bar{\delta}_{y} \Phi\right)^{-}}{q_{j, k-1}}\left[h\left(q_{j, k-1}, q_{j k}\right)+\rho(s) s\right]+q_{j k} \bar{\delta}_{y}\left(\frac{\left(\vec{\delta}_{y} \Phi\right)^{-}}{q}\right) \\
& \quad \leqslant q_{j k} \bar{\delta}_{y}\left(\frac{\left(\vec{\delta}_{y} \Phi\right)^{-}}{q}\right)=q\left(1-\chi\left(\sin \theta^{\mathrm{IV}}\right)\right) \bar{\delta}_{y}\left(\sin \theta^{\mathrm{IV}}\right)
\end{aligned}
$$

(for the usual E flux reason).

We now add

$$
-\left(1-\chi\left(\sin \theta^{\mathrm{IV}}\right)\right) \overrightarrow{\boldsymbol{\delta}}_{y}\left(\left(q_{j k} \cos \theta^{\mathrm{IV}}\right) \theta_{j k-1}^{\mathrm{IV}}\right)
$$

to (5.10) arriving at

$$
\begin{aligned}
(1-\chi & \left.\left(\sin \theta^{\mathrm{IV}}\right)\right) q\left[\bar{\delta}_{y} \sin \theta^{\mathrm{IV}}-\left(\cos \theta^{\mathrm{IV}}\right) \bar{\delta}_{y} \theta^{\mathrm{IV}}\right] \\
& -\left(1-\chi\left(\sin \theta^{\mathrm{IV}}\right)\right) \theta^{\mathrm{IV}} \vec{\delta}_{y}\left(q \cos \theta^{\mathrm{IV}}\right) \\
= & -q\left|\sin \theta^{\mathrm{IV}}\right| \frac{\left(1-\cos \left(\Delta y \bar{\delta}_{y} \theta^{\mathrm{IV}}\right)\right)}{\Delta y} \\
& +\left(1-\chi\left(\sin \theta^{\mathrm{IV}}\right)\right) \cos \theta^{\mathrm{IV}}\left(\frac{\sin \left(\Delta y \bar{\delta}_{y} \theta^{\mathrm{IV}}\right)}{\Delta y}-\overline{\boldsymbol{\delta}}_{y} \theta^{\mathrm{IV}}\right) \\
& -\left(1-\chi\left(\sin \theta^{\mathrm{IV}}\right)\right) \theta^{\mathrm{IV}} \vec{\delta}_{y}\left(q \cos \theta^{\mathrm{IV}}\right) \\
\leqslant & -\left(1-\chi\left(\sin \left(\theta^{\mathrm{IV}}\right)\right) \theta^{\mathrm{IV}} \vec{\delta}_{y}\left(q \cos \theta^{\mathrm{IV}}\right)\right) \text { if }\left|\Delta y \overline{\boldsymbol{\delta}}_{y} \boldsymbol{I}^{\mathrm{IV}}\right| \text { is small enough. }
\end{aligned}
$$

Adding (5.5), (5.7), (5.9), (5.11) gives us (5.3) if we can prove

$$
\begin{aligned}
& \chi\left(\cos \theta^{\mathrm{I}}\right) \theta^{\mathrm{I}} \hat{\delta}_{x}\left(q \sin \theta^{\mathrm{I}}\right)-\chi\left(\sin \theta^{\mathrm{III}}\right) \theta^{\mathrm{III}} \hat{\delta}_{y}\left(q \cos \theta^{\mathrm{III}}\right) \\
& +\left(1-\chi\left(\cos \theta^{\mathrm{II}}\right)\right) \theta^{\mathrm{II}} \vec{\delta}_{x}\left(q \sin \theta^{\mathrm{II}}\right)-\left(1-\chi\left(\sin \theta^{\mathrm{IV}}\right)\right) \theta^{\mathrm{IV}} \vec{\delta}_{y}\left(q \cos \theta^{\mathrm{IV}}\right)=0 .
\end{aligned}
$$

We show this by considering four cases:

(1) $\cos \theta^{\mathrm{I}}>0, \sin \theta^{\mathrm{III}}>0$.

This implies that $\theta^{\mathrm{I}}=\theta^{\mathrm{III}}$ and $q \sin \theta^{\mathrm{I}}=\overleftarrow{\delta}_{y} \Phi, q \cos \theta^{\mathrm{III}}=\overleftarrow{\delta}_{x} \Phi$.

(2) $\cos \theta^{\mathrm{I}}>0, \sin \theta^{\mathrm{IV}}<0$.

This implies that $\theta^{\mathrm{I}}=\theta^{\mathrm{IV}}$ and $q \sin \theta^{\mathrm{I}}=\vec{\delta}_{y} \Phi, q \cos \theta^{\mathrm{IV}}=\bar{\delta}_{x} \Phi$.

(3) $\cos \theta^{\mathrm{II}}<0, \sin \theta^{\mathrm{III}}>0$.

This implies $\theta^{\mathrm{II}}=\theta^{\mathrm{III}}$ and $q \cos \theta^{\mathrm{III}}=\vec{\delta}_{x} \Phi, q \sin \theta^{\mathrm{II}}=\bar{\delta}_{y} \Phi$.

(4) $\cos \theta^{\mathrm{II}}<0, \sin \theta^{\mathrm{IV}}<0$.

This implies $\theta^{\mathrm{II}}=\theta^{\mathrm{IV}}$ and $q \cos \theta^{\mathrm{IV}}=\vec{\delta}_{x} \Phi, q \sin \theta^{\mathrm{II}}=\vec{\delta}_{y} \Phi$.

In all cases, Eq. (5.12) is now easily verified. 
VI. Numerical Results and Computational Comments. In the previous sections, we considered the scheme approximating (2.1)-(2.4) in Cartesian coordinates. Real computations involve body-fitted and in general, nonorthogonal coordinates. Fortunately, our schemes transform quite simply under such coordinate changes, even in three space dimensions.

Given a change of variables, we proceed as follows: Let

$$
\zeta=\zeta(x, y, z), \quad \eta=\eta(x, y, z), \quad \xi=\xi(x, y, z) .
$$

The potential equation becomes

$$
\left(\frac{\rho U}{J}\right)_{\zeta}+\left(\frac{\rho V}{J}\right)_{\eta}+\left(\frac{\rho W}{J}\right)_{\xi}=0
$$

For simplicity of exposition, we define

$$
\begin{array}{lll}
U=U_{1}, & V=U_{2}, & W=U_{3}, \\
x=x_{1}, & y=x_{2}, & z=x_{3}, \\
\zeta=X_{1}, & \eta=X_{2}, & \xi=X_{3} .
\end{array}
$$

Here

$$
U_{i}=\sum_{j=1}^{3} a_{i j} \Phi_{X_{j}}, \quad i=1,2,3,
$$

with

$$
\begin{gathered}
a_{i j}=\sum_{k=1}^{3} \frac{\partial X_{i}}{\partial x_{k}} \frac{\partial X_{j}}{\partial x_{k}}, \quad i, j=1,2,3 \\
a_{i j}=\mathscr{J} \mathscr{J}^{*}, \\
\rho=\left[1-\frac{\gamma-1}{2} M_{\infty}^{2}\left(U_{1} \Phi_{X_{1}}+U_{2} \Phi_{X_{2}}+U_{3} \Phi_{X_{3}}-1\right)\right]^{1 /(\gamma-1)} \\
=\left[1-\frac{\gamma-1}{2} M_{\infty}^{2}\left(q^{2}-1\right)\right]^{1 /(\gamma-1)}, \\
q^{2}=\sum_{i, j=1}^{3} \Phi_{X_{i}} a_{i j} \Phi_{X_{j}}, \\
J=\frac{\partial\left(X_{1}, X_{2}, X_{3}\right)}{\partial\left(x_{1}, x_{2}, x_{3}\right)}=\operatorname{det}\left\{\frac{\partial X_{i}}{\partial x_{j}}\right\}=\operatorname{det}[\mathscr{J}] .
\end{gathered}
$$

We proceed numerically as follows:

Approximate

$$
q^{2}=\sum_{i, j=1}^{3}\left(\overleftarrow{\delta}_{X_{i}} \Phi\right)^{+} a_{i j}\left(\overleftarrow{\delta}_{X_{j}} \Phi\right)^{+}+\sum_{i, j=1}^{3}\left(\vec{\delta}_{X_{i}} \Phi\right)^{-} a_{i j}\left(\vec{\delta}_{X_{j}} \Phi\right)^{-}
$$

This defines $q, \rho$, and $a$ on the grid.

Let $q^{*}, p^{*}$ and $(\rho q)_{-}$be as before. Then, the basic space differencing for a given $\mathrm{E}$ flux is

$$
\begin{aligned}
\frac{\partial}{\partial X_{1}}\left(\frac{\rho U_{1}}{J}\right)= & \frac{\partial}{\partial \zeta}\left(\frac{\rho U}{J}\right) \approx \vec{\delta}_{X_{1}}\left[\frac{\left(\sum_{j=1}^{3} a_{1 j} \overleftarrow{\delta}_{X_{i}} \Phi\right)^{+}}{J q}\left[-h\left(q_{j k l}, q_{j-1, k, l}\right)\right]\right] \\
& +\overleftarrow{\delta}_{X_{i}}\left[\frac{\left(\sum_{j=1}^{3} a_{1 j} \vec{\delta}_{X_{i}} \Phi\right)^{-}}{J q}\left[-h\left(q_{j k l}, q_{j+1, k, l}\right)\right]\right] .
\end{aligned}
$$

The $X_{2}$ and $X_{3}$ differencing is analogous. 


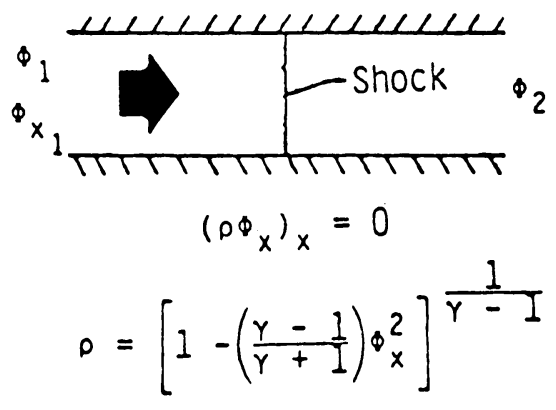

Figure 1 (a)

Typical one-dimensional flow

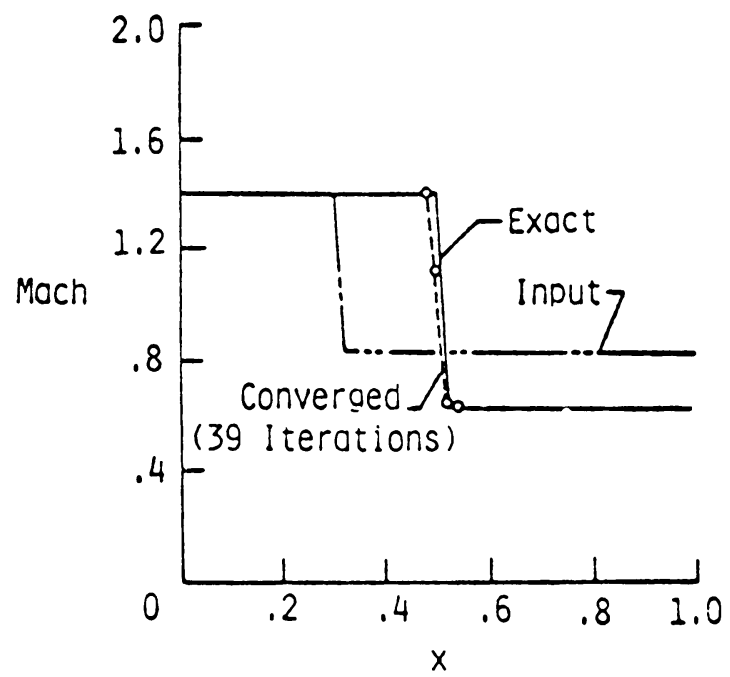

Figure 1 (b)

Calculation of one-dimensional compression shock

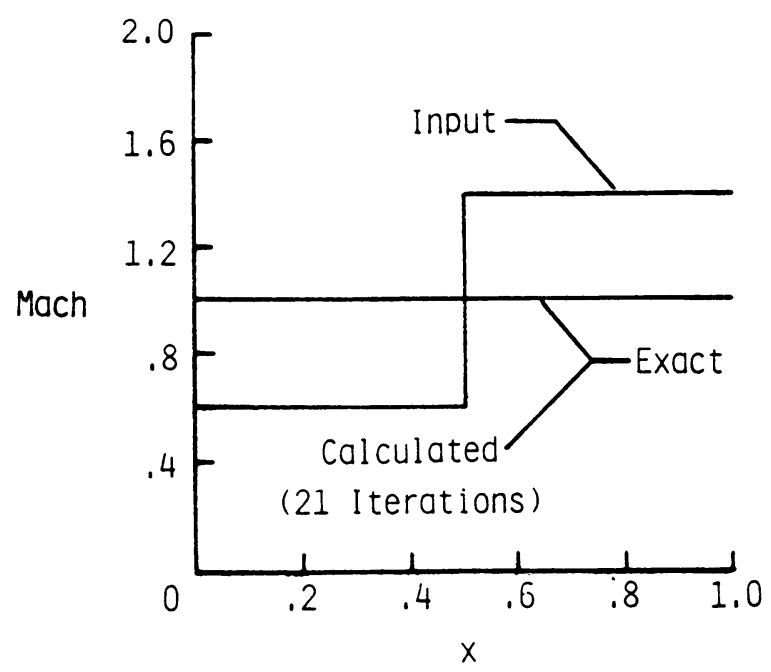

Figure 1 (c)

Elimination of one-dimensional expansion shock 
If we use the E.O. flux, we have

$$
\begin{aligned}
\frac{\partial}{\partial X_{1}}\left(\frac{\rho U_{1}}{J}\right)= & \frac{\partial}{\partial \zeta}\left(\frac{\rho U}{J}\right) \\
\approx & \vec{\delta}_{X_{i}}\left[\frac{\left(\sum_{j=1}^{3} a_{1 j} \overleftarrow{\delta}_{X_{j}} \Phi\right)^{+}}{J q}\left[\rho q-\Delta X_{1} \overleftarrow{\delta}_{X_{1}}(\rho q)_{-}\right]\right] \\
& +\overleftarrow{\delta}_{X_{i}}\left[\frac{\left(\sum_{j=1}^{3} a_{1 j} \vec{\delta}_{X_{j}} \Phi\right)^{-}}{J q}\left[\rho q+\Delta X_{1} \vec{\delta}_{X_{1}}(\rho q)_{-}\right]\right]
\end{aligned}
$$

The $X_{2}$ and $X_{3}$ differencing is analogous.

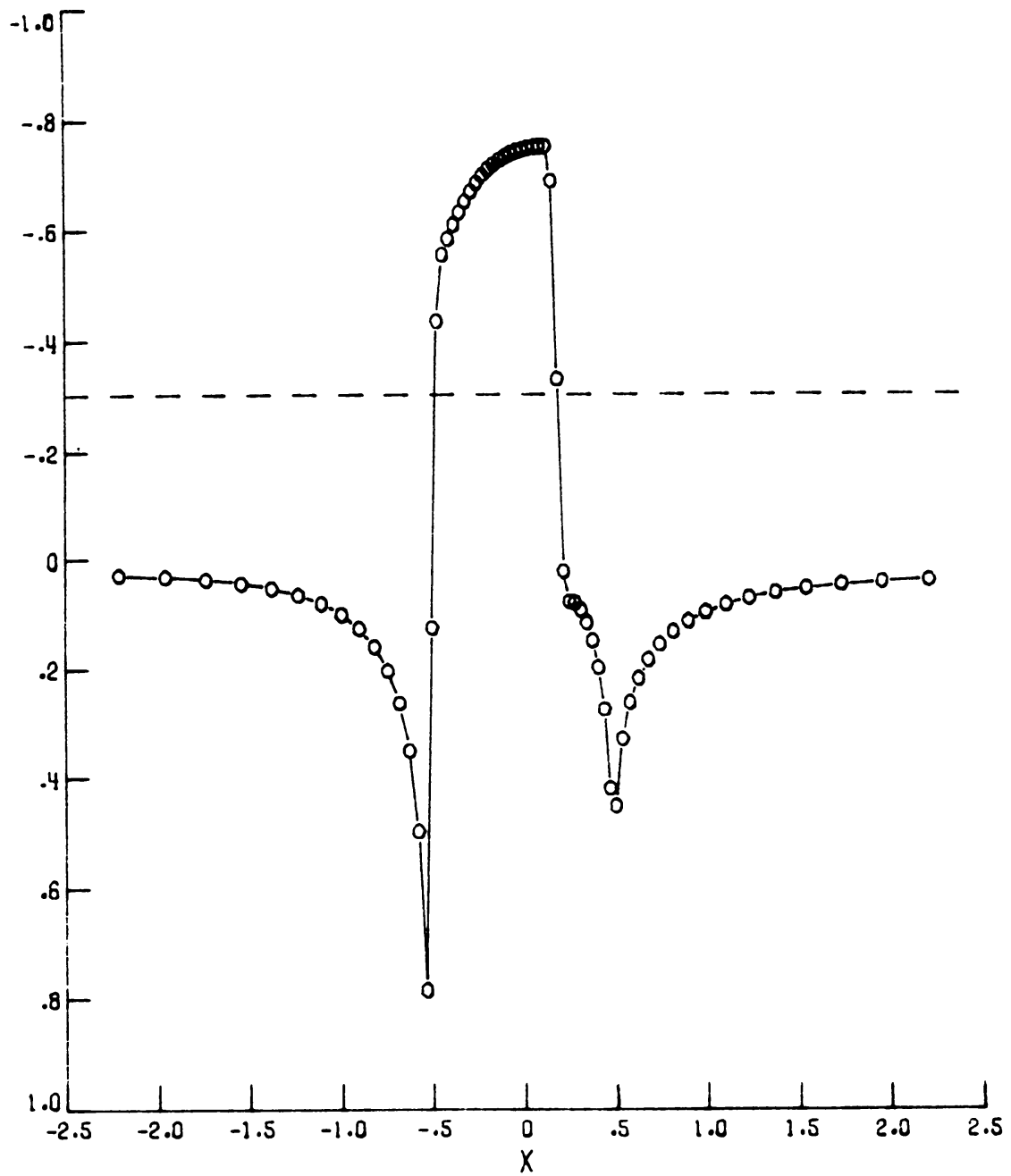

FIGURE 2

Pressure distribution on an NACA 0012 at $M=0.85$

and 0 degrees angle of attack 
We first perform a simple test calculation by solving the one-dimensional FP in two cases. First we input boundary conditions which should produce a valid compression shock, and take an initial guess which has to move to the correct steady state. We use Newton's method directly and get convergence in 39 iterations, as shown in Figure 1(b).

In Figure 1(c), we reverse the boundary and initial data, eliminate the expansion shock, and converge to the correct steady state in 21 iterations, again using Newton's method.

In Figure 2, we solve FP in two dimensions for a NACA 0012 airfoil for free stream $M=.85$. We plot the converged $c_{p}$ at the upper surface. Here we used Cartesian coordinates and our new switches in the $x$ direction only.

Figure 3 shows the convergence history in this case.

In Figure 4, we show the results using generalized coordinates and the switches in the streamwise direction only.

Figure 5 gives the convergence history in this case.

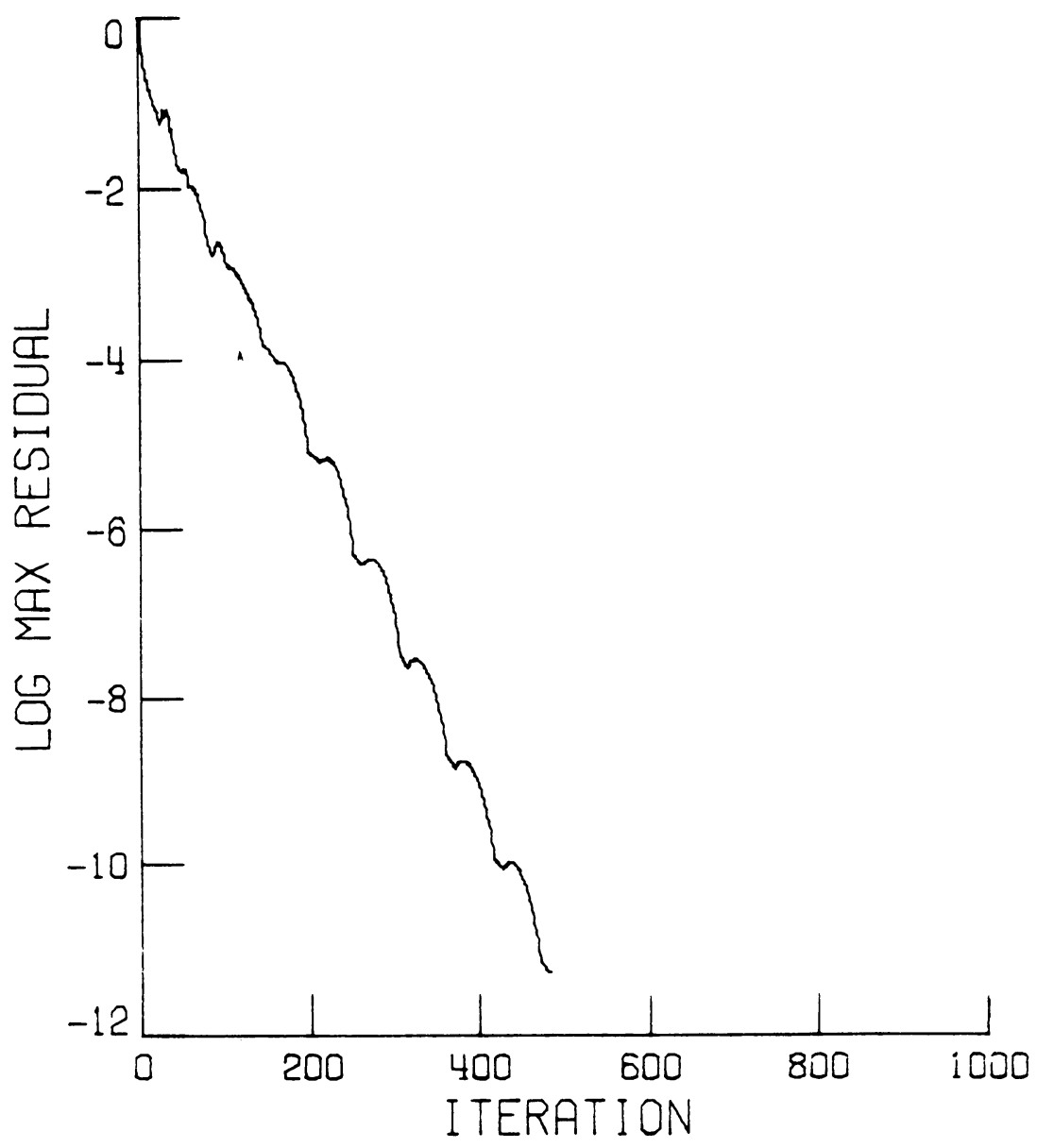

FIGURE 3

Convergence history for an NACA 0012 at $M=0.85$

and 0 degrees angle of attack 


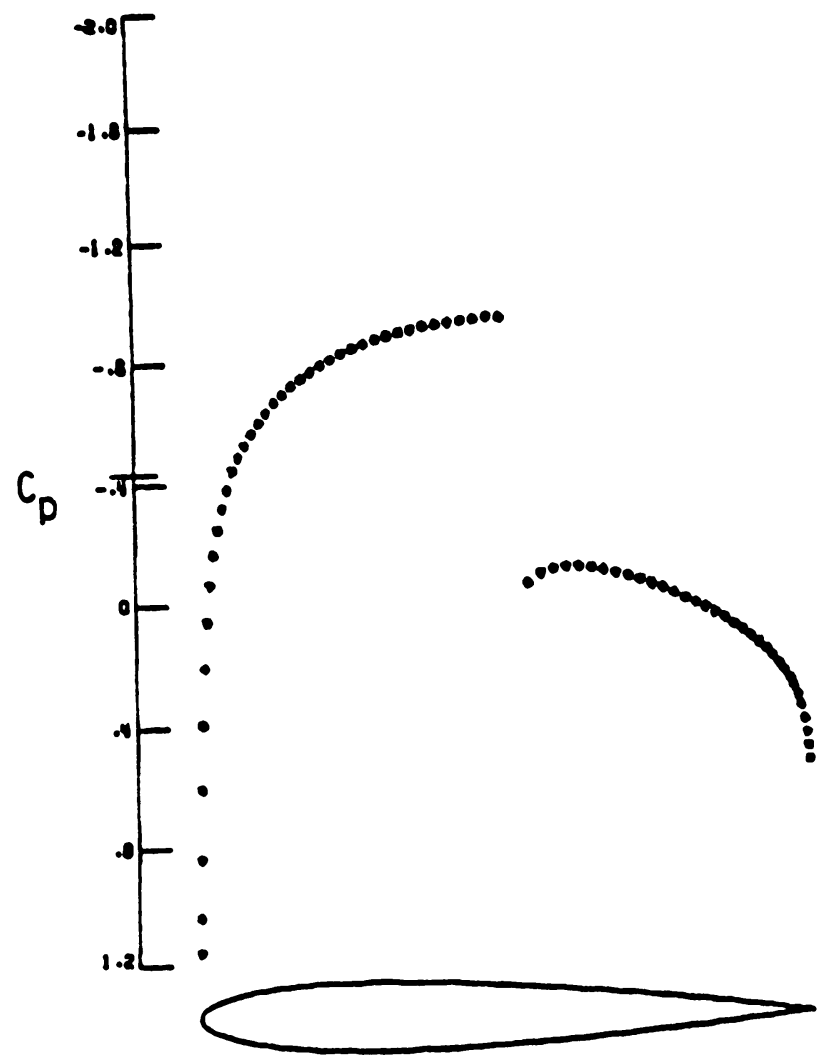

Figure 4

Pressure distribution on an NACA 0012 at $M=0.80$ and 0 degrees angle of attack 


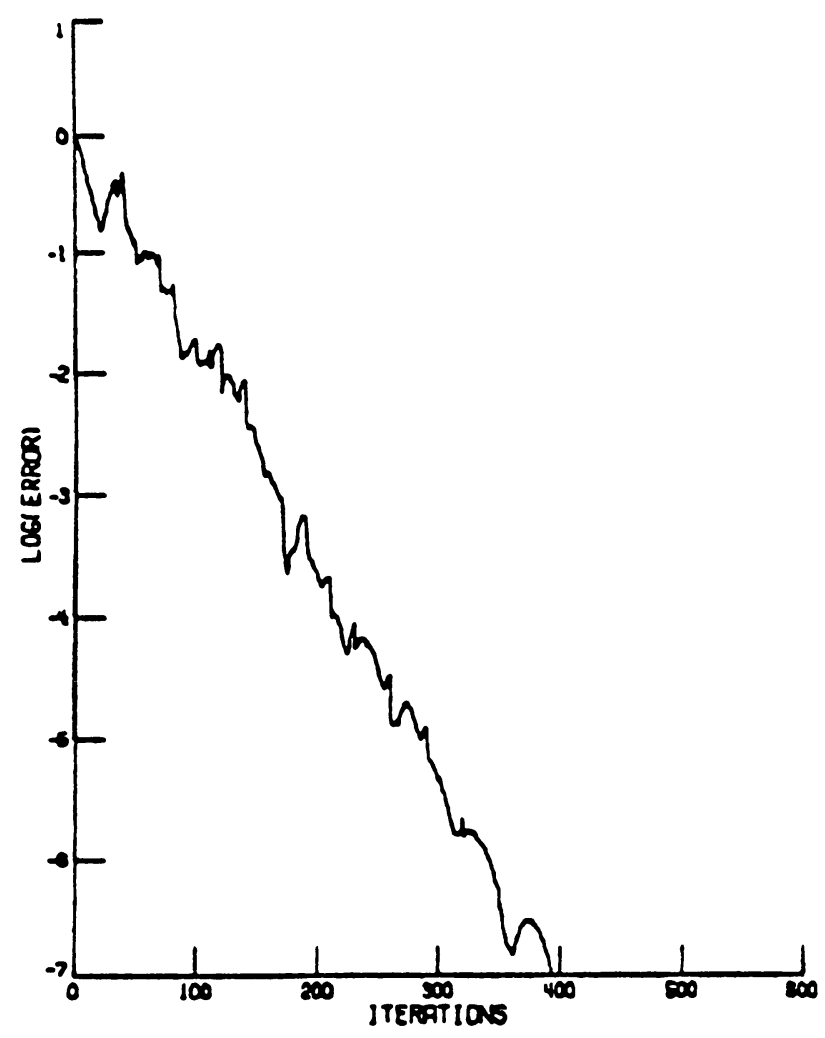

FIGURE 5

Convergence history for an NACA 0012 at $M=0.80$ and 0 degrees angle of attack 


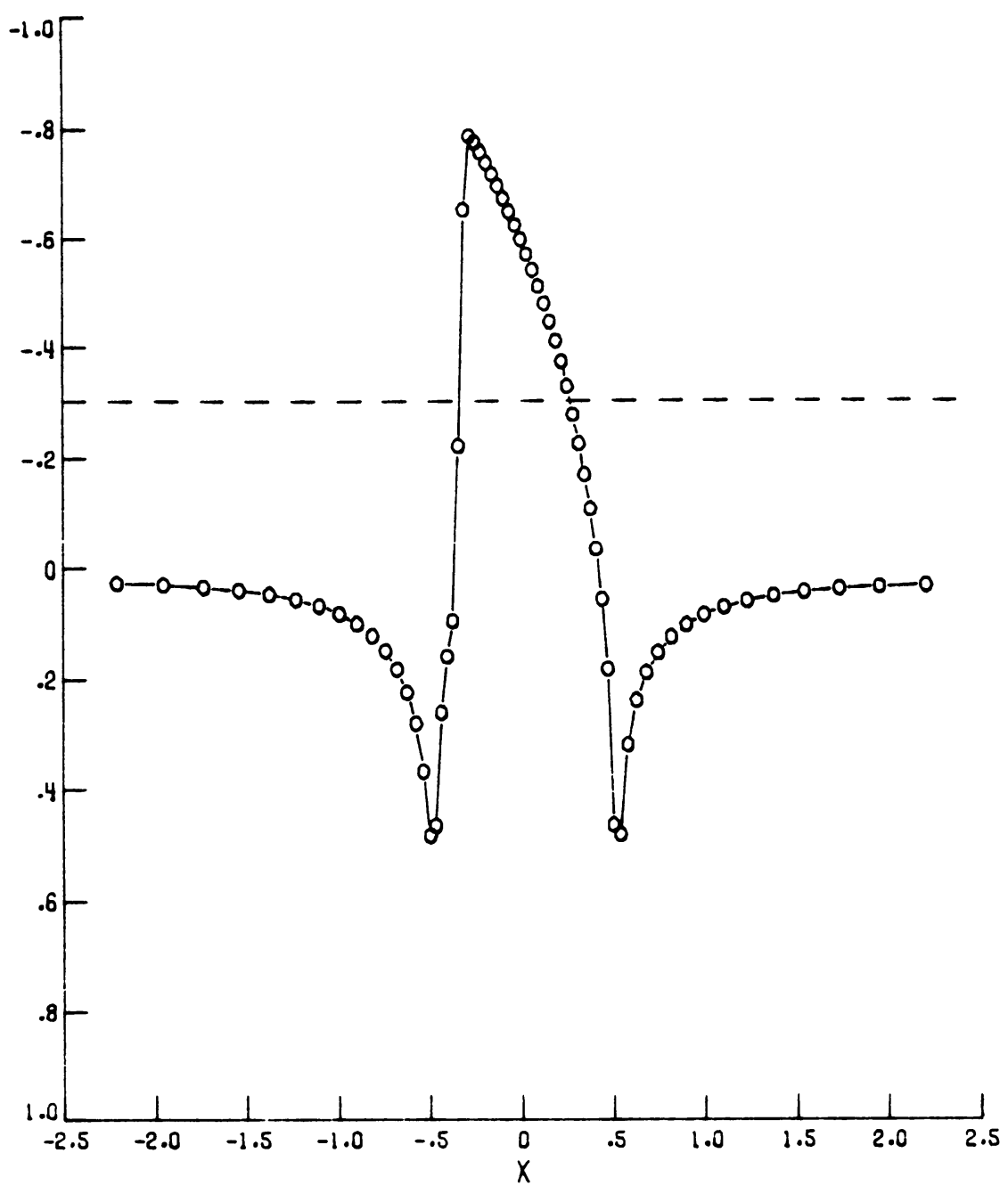

FIGURE 6

Reverse flow input for a 10 percent thick parabolic arc airfoil at $M=0.85$ and 0 degrees angle of attack 


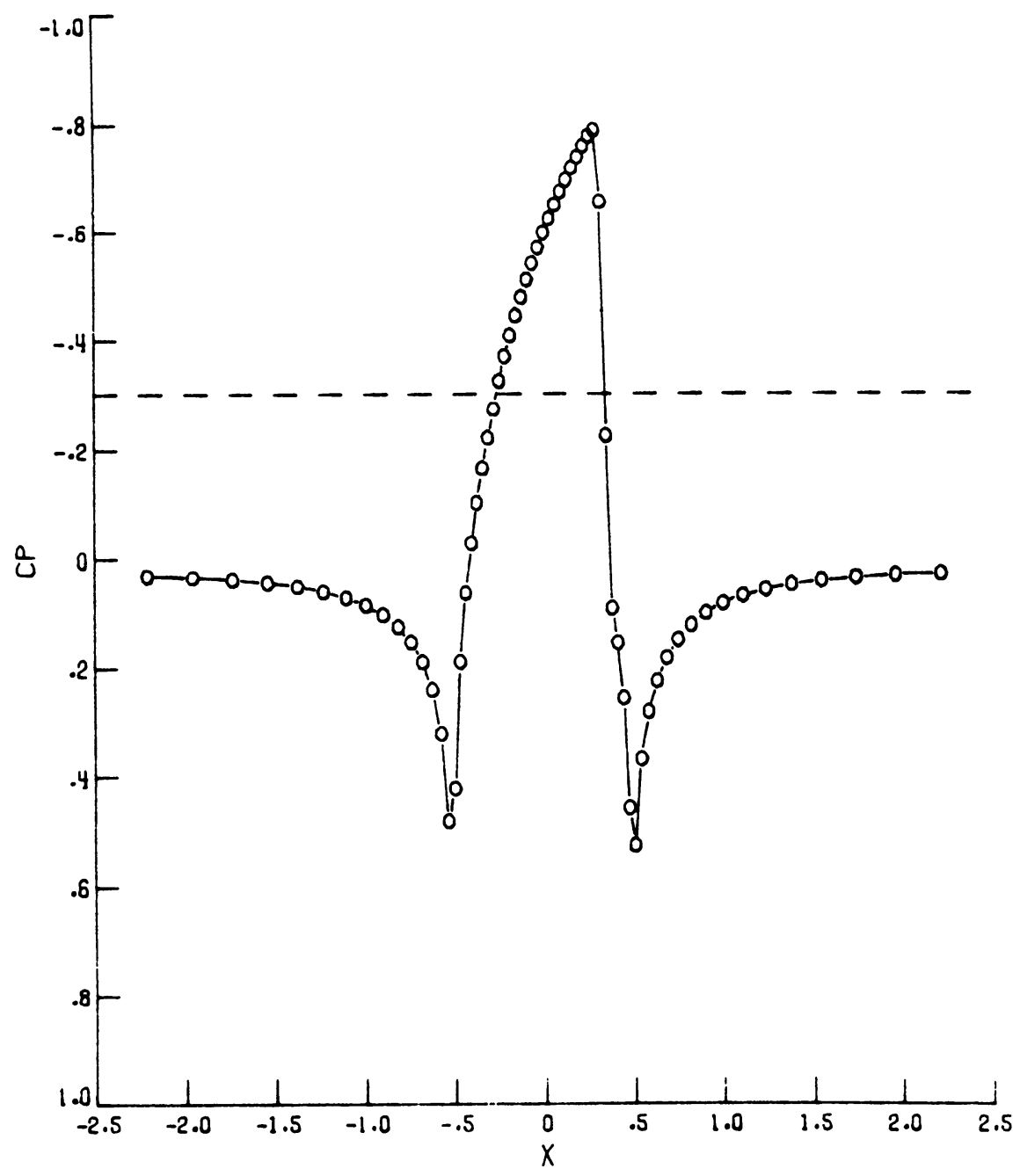

FIGURE 7

Calculated pressure distribution on a 10 percent thick parabolic arc airfoil at $M=0.85$ and 0 degrees angle of attack 


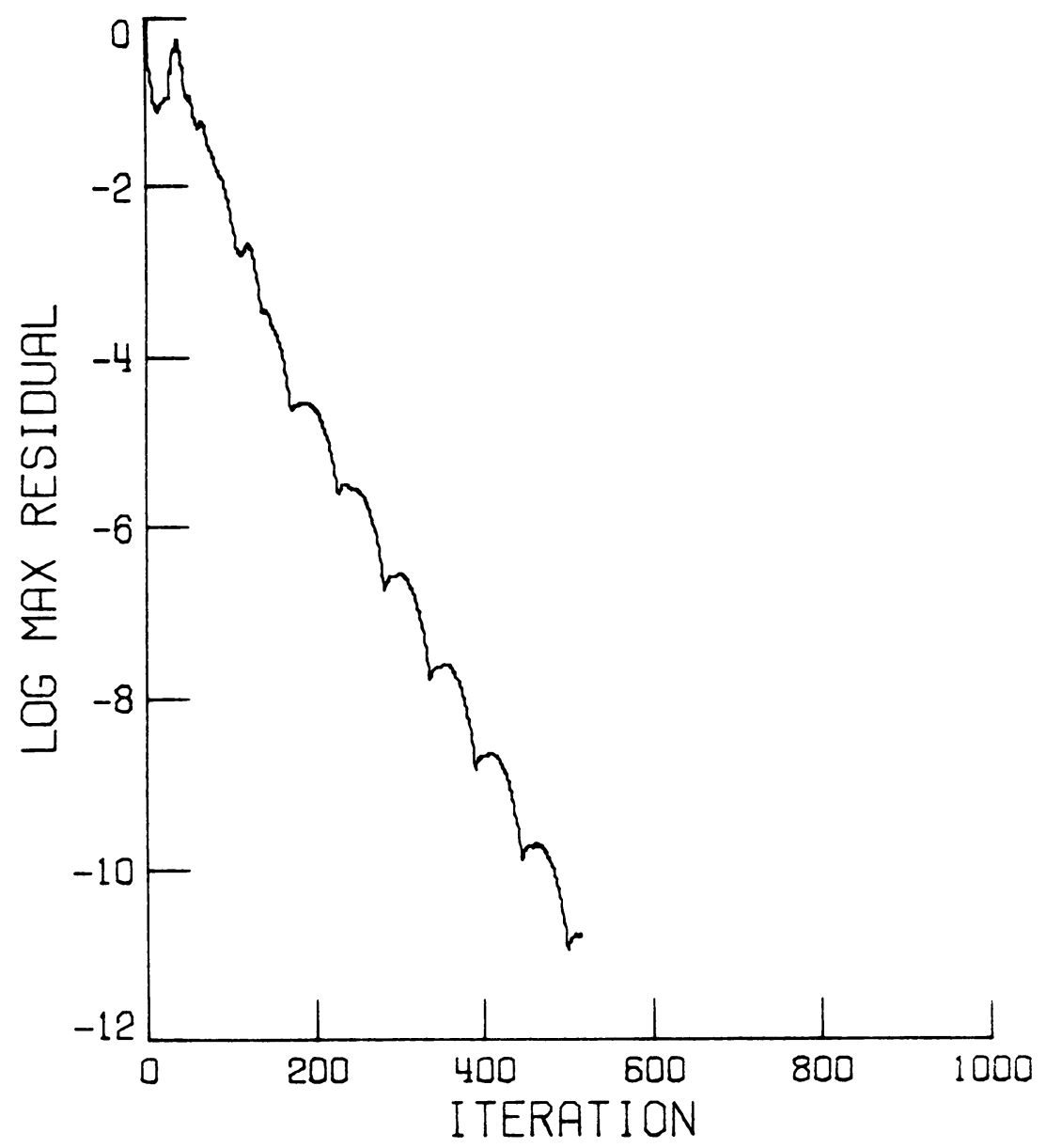

FIGURE 8

Convergence history for a 10 percent thick parabolic arc airfoil at $M=0.85$ and 0 degrees angle of attack 


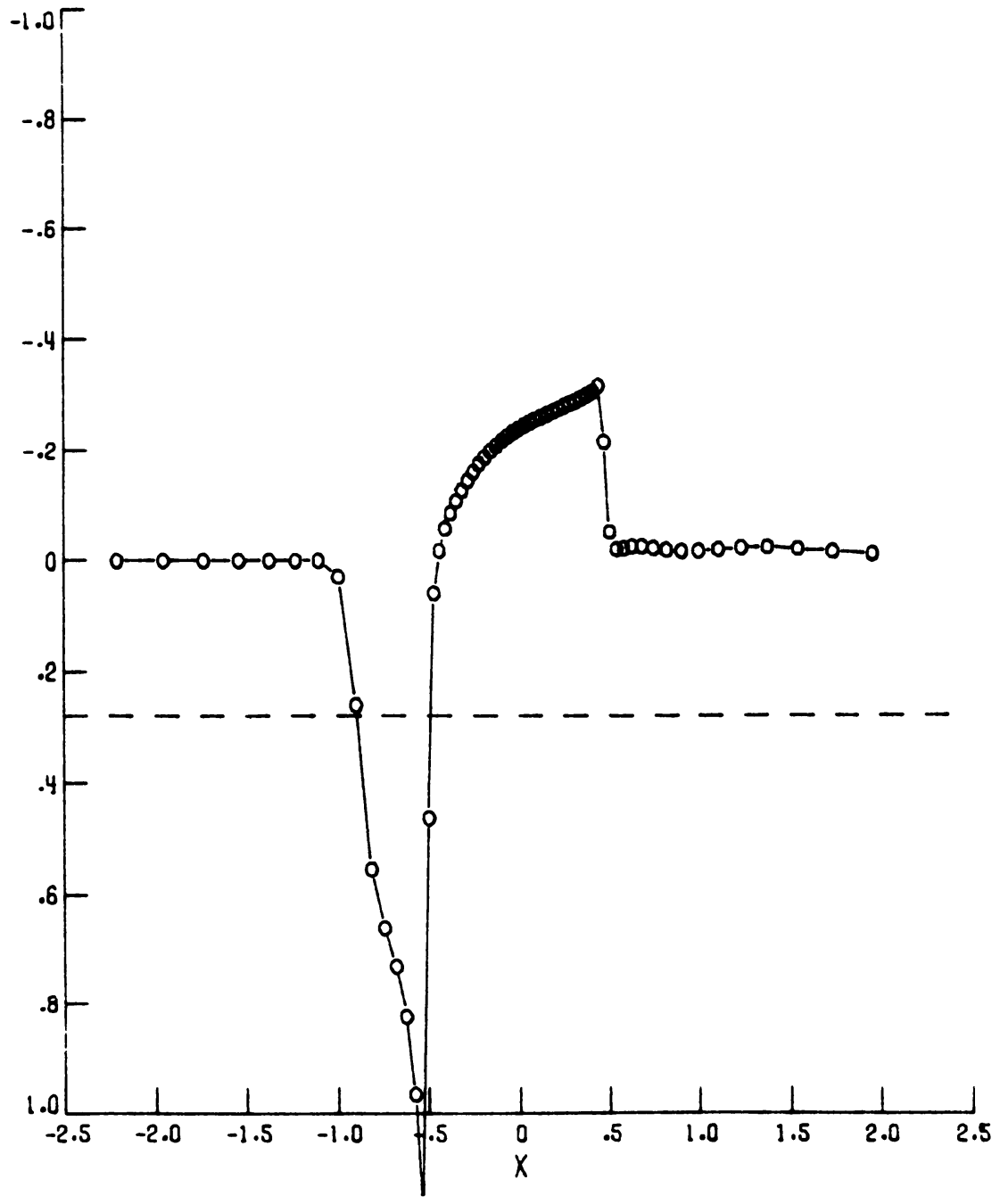

Figure 9

Pressure distribution on an NACA 0012 at $M=1.2$ and 0 degrees angle of attack 
Next we consider a $10 \%$ parabolic arc, with $M=.85$, and input an exact solution to reverse flow, i.e., replace $x$ by $-x$ in the true steady solution. This nonphysical solution evolves according to our algorithm using the switching in the $x$ direction with Cartesian coordinates. The correct steady state emerged, see Figures 6, and 7, with Figure 8 giving the convergence history.

Finally, we took the NACA 0012 airfoil, with $M=1.2$, using our switching in the $x$ direction with Cartesian coordinates. The $c_{p}$ upper surface pressure plot gives the crisp fishtail shock seen in Figure 9.

Mathematics Department

University of California

Los Angeles, California 90024

NASA Langley Research Center

Hampton, Virginia 23665

1. J. W. BoERSTOEL, “A multigrid algorithm for steady transonic potential flows around airfoils using Newton's iteration,” J. Comput. Phys., v. 48, 1982, pp. 313-343.

2. S. R. Chakravarthy \& S. Osher, "High resolution applications of the Osher upwind scheme for the Euler equations," AIAA Computational Fluid Dynamics Proceedings, Danvers, Mass., 1983, pp. 363-372.

3. R. Chipman \& A. Jameson, Fully Conservative Numerical Solutions for Unsteady Irrotational Flow About Airfoils, AIAA paper 79-1555, 1979.

4. P. Colella \& P. R. Woodward, The Piecewise-Parabolic Method (PPM) for Gas Dynamical Simulations, LBL report \#14661, July, 1982.

5. R. J. DiPerna, "Decay of solutions of hyperbolic systems of conservation laws with a convex extension," Arch. Rational Mech. Anal., v. 64, 1977, pp. 1-92.

6. A. EBERLE, Eine method finiter elements berechnung der transsonicken potential-strimung un profile, MBB Berech Nr. UFE 1352(0), 1977.

7. J. W. Edwards, R. M. Bennett, W. Whitlow, JR. \& D. A. Seidel, Time Marching Transonic Flutter Solutions Including Angle-of-Attack Effects, AIAA Paper 82-0685, New Orleans, Louisiana, 1982.

8. B. ENGQUIST \& S. OSHER, "Stable and entropy satisfying approximations for transonic flow calculations," Math. Comp., v. 34, 1980, pp. 45-75.

9. B. ENGQUIST \& S. OSHER, “Discrete shocks and upwind schemes.” (In preparation.)

10. K. O. FrIEDRichs \& P. D. LAX, "Systems of conservation laws with a convex extension," Proc. Nat. Acad. Sci. U.S.A., v. 68, 1971, pp. 1686-1688.

11. S. K. Godunov, "A finite difference method for the numerical computation of discontinuous solutions of the equations of fluid dynamics," Mat. Sb., v. 47, 1959, pp. 271-290. (Russian)

12. P. M. Goorjian, M. Meagher \& R. VAn Buskirk, Monotone Implicit Algorithms for the Small Disturbance and Full Potential Equation Applied to Transonic Flow, AIAA paper 83-0371, 1983.

13. P. M. GoorJiAN \& R. VAN BuSKIRK, Implicit Calculations of Transonic Flow Using Monotone Methods, AIAA Paper 81-331, St. Louis, Missouri, 1981.

14. M. M. Hafez, E. M. Murman \& J. E. South, Artificial Compressibility Methods for Numerical Solution of Transonic Full Potential Equation, AIAA paper 78-1148, Seattle, Wash., 1978.

15. M. Hafez, W. Whitlow, JR. \& S. OsheR, "Improved finite difference schemes for transonic potential calculations," AIAA Paper 84-0092, Reno, Nevada, 1984.

16. A. Harten, "High resolution schemes for conservation laws," J. Comput. Phys., v. 49, 1983, pp. 357-393.

17. T. L. Holst \& W. F. Ballhaus, "Fast, conservative schemes for the full potential equation applied to transonic flows," AIAA J., v. 17, 1979, pp. 145-152.

18. A. JAMESON, "Numerical solutions of nonlinear partial differential equations of mixed type," Numerical Solutions of Partial Differential Equations III, Academic Press, New York, 1976, pp. 275-320.

19. A. JAMESON, "Transonic potential flow calculations using conservative form," AIAA Second Computational Fluid Dynamics Proceedings, Hartford, Conn., 1975, pp. 148-155.

20. P. D. LAX, Hyperbolic Systems of Conservation Laws and the Mathematical Theory of Shock Waves, SIAM Regional Conf. Series Lectures in Appl. Math., Vol. 11, 1972. 
21. E. MuRman, “Analysis of embedded shockwaves calculated by relaxation methods," AIAA J., v. 12 , 1974 , pp. 626-633.

22. S. OSHER, Numerical Solution of Singular Perturbation Problems and Hyperbolic Systems of Conservation Laws, North-Holland Math. Stud. No. 47 (O. Axelsson, L. S. Frank and A. van der Sluis, eds.), 1981, pp. 179-205.

23. S. OSHER, "Riemann solvers, the entropy condition, and difference approximations," SIAM J. Numer. Anal., v. 21, 1984, pp. 217-235.

24. S. Osher \& S. Chakravarthy, "High resolution schemes and the entropy condition," SIAM J. Numer. Anal., v. 21, 1984, pp. 955-984.

25. P. L. RoE, “Approximate Riemann solvers, parameter vectors, and difference schemes," J. Comput. Phys., v. 43, 1981, pp. 357-372.

26. Ph. Morice \& H. Vivand, "Équations de conservation et condition d'irréversibilité pour les écoulements transsoniques potentiels," C. R. Acad. Sci. Paris Sér. A-B, v. 291, 1980, pp. B235-B238.

27. B. VAN LEER, "Towards the ultimate conservative difference scheme, II. Monotonicity and conservation combined in a second - order scheme," J. Comput. Phys., v. 14, 1974, pp. 361-370.

28. B. VAN LEER, "Towards the ultimate conservative difference scheme V. A second order sequel to Godunov's method," J. Comput. Phys., v. 32, 1979, pp. 101-136.

29. B. VAN LEER, "On the relation between the upwind-differencing schemes of Godunov, Engquist-Osher, and Roe," SIAM J. Sci. Statist. Comput., v. 5, 1984, pp. 1-20.

30. L. C. Wellford, JR. \& M. M. Hafez," A finite element first-order equation for the small disturbance transonic flow problem," Comput. Methods Appl. Mech. Engrg., v. 22, 1980, pp. 161-186.

31. P. D. Lax \& B. Wendroff, "Systems of conservation laws," Comm. Pure Appl. Math., v. 23, 1960, pp. 217-237. 